\title{
Analytical Solutions for Impulsive Elliptic Out-of-Plane Rendezvous Problem via Primer Vector Theory
}

\author{
Romain Serra, Denis Arzelier and Aude Rondepierre
}

\begin{abstract}
This paper focuses on the fixed-time minimum-fuel out-of-plane rendezvous between close elliptic orbits of an active spacecraft, with a passive target spacecraft, assuming a linear impulsive setting. It is shown that the out-of-plane elliptic relative dynamics are simple enough to allow for an analytical solution of the problem reviewed. Indeed, the approach relies on the primer vector theory by writing down and directly solving the optimality necessary conditions. After analyzing the characteristics of the dynamics of the optimal primer vector candidates, the complete analytical optimal solution is obtained for arbitrary durations of the rendezvous and arbitrary boundary conditions.
\end{abstract}

Index Terms-Orbital rendezvous, fuel optimal space trajectories, primer vector theory, impulsive maneuvers, linear equations of motion.

\section{INTRODUCTION}

For the next years, there will be an increasing demand for the efficient execution of the autonomous rendezvous between an active chaser spacecraft and a passive target spacecraft. Therefore, new challenges are met when designing appropriate guidance schemes for achieving autonomous far range rendezvous on highly elliptical orbits. Autonomy means that the simplicity of onboard implementation while preserving optimality in terms of fuel consumption, is a fundamental feature of the proposed solution. Hence, obtaining an analytical closed-form solution for these types of problems is of paramount interest and induces, in general, a more efficient and rapid technological solution.

Here, the fixed-time linearized minimum-fuel impulsive rendezvous problem, as defined in [1], [2], is studied. The impulsive approximation for the thrust means that instantaneous velocity increments are applied to the chaser whereas its position is continuous. The focus of the paper is on the elliptic out-of-plane (OOP) rendezvous problem for which no complete optimal solution exists to the best of our knowledge. When the eccentricity of the reference orbit is equal to 0 , the out-of-plane rendezvous problem amounts to solving an optimal impulsive control problem for a simple harmonic oscillator. A complete characterization of the optimal solutions for this problem has been given by Prussing in [3]. In [1],

R. Serra was with LAAS-CNRS, 7 avenue du colonel Roche, F-31400 Toulouse, F-31400 Toulouse, France. Present address: Department of Mechanical and Aerospace Engineering, James Weir Building, 75 Montrose Street, Glasgow G1 1XJ, United Kingdom.

D. Arzelier is with LAAS-CNRS, 7 avenue du colonel Roche, F-31400 Toulouse, F-31400 Toulouse, France

A. Rondepierre is with IMT, University of Toulouse, 118 route de Narbonne, F-31400 Toulouse, France a complete solution for the circular coplanar case has been given for every possible boundary condition for a duration of rendezvous of $2 \pi$. As the relative motion between two vehicles in highly elliptic orbits differs significantly from the relative motion seen in circular rendezvous, the solution of the elliptic problem is much more complicated and more recent efforts have investigated this particular problem (see [4] and [5] and the references therein). As mentioned in [5], the previous approaches, including the most recent ones, all suffer from different shortcomings: limitation to the circular or quasicircular cases, numerical complexity of the solution and/or lack of comprehensiveness. For instance, the results given in [4] are limited to the 1-impulse strategies while those of [5] rely on an iterative numerical process highly dependent upon the choice of an initial guess.

The contribution of the paper is to give a complete analytical solution of the problem of fuel-optimal impulsive linearized out-of-plane rendezvous when the reference orbit is elliptic, whatever the duration of the rendezvous and for all possible initial and terminal conditions. Despite the number of cases to be discussed (eight cases strongly depending on the duration of the rendezvous and the initial and final conditions), this analytical solution is actually very simple, explicitly expressed in terms of the data of the rendezvous problem and does not require time-consuming computations to be obtained. This is a key point for the onboard implementation and the autonomy of futures missions.

The solutions are obtained via the analysis of the optimal conditions expressed in terms of the primer vector as in [1] and [3]. When eccentricity of the reference orbit is not equal to 0 , the primer vector exhibits particular features that may be analyzed and which provide the basis for the derivation of the optimal solution. It is shown that the nature of the optimal solutions (number and locations of the optimal thrusts) strongly depends upon the duration of the rendezvous. Indeed, three ranges of duration are identified and the analytical closed-form solutions are given for each, with their conditions of validity expressed in terms of the eccentricity, the boundary conditions, initial and final anomaly of the rendezvous problem. Every result is rigorously proved by relying on the necessary and sufficient optimality conditions and working out these conditions to exhibit the optimal solutions. In addition, it is shown that the designer has extra degrees of freedom when the rendezvous lasts more than one revolution. Indeed, the optimal consumption may be spread over a maximum number of impulses that may be greater than the known 
upper-bound given by Neustadt [6] on the optimal number of impulses. By optimal number of impulses, it is meant the minimum number of impulses necessary to obtain the minimum fuel consumption for some instance of the linear fixed-time rendezvous problem. As a side result, it is also shown when considering some degenerate cases where the Lagrange multipliers involved in the computation of the primer vector are not unique and are underdetermined, that it is always possible to choose a particular solution for which an optimal primer vector will be completely defined. Finally, two realistic examples illustrate these results. Note that a preliminary version of this paper has been presented at the IFAC World Congress [7] in which results are stated without any proof and degenerate cases are not explored.

The outline of the paper is as follows. In the next section, the problem formulation is given. The Section III. recalls the optimality conditions expressed in terms of conditions on the primer vector. The properties of primer vector candidates for optimality are analyzed in Section IV The complete set of optimal solutions for the minimum-fuel out-of-plane linearized rendezvous problem is given in Section $\mathrm{V}$. As there are many cases to be considered, two tables are given in this section. The first one summarizes the 8 different possible solutions with their velocity increments, the location of the optimal impulses while the second gives the conditions to be met for each type of solution depending on the duration of the rendezvous. Section VI is dedicated to the presentation of two realistic numerical examples illustrating different cases. Due to space constraints, only the proof of the first proposition is given in the Appendix. The other proofs follow the same lines and may be found in the technical note [8].

The main notations are defined hereafter: the vectors are noted with an arrow as in $\vec{z}_{f}$ while matrices are in boldface. as in $\boldsymbol{\Phi}$.

\section{Notations:}

- $a$ : semi-major axis, $\mathrm{m}$

- $e$ : eccentricity

- $\theta$ : true anomaly, rad

- $\boldsymbol{\Phi}\left(\theta, \theta_{0}\right)=\boldsymbol{\phi}(\theta) \boldsymbol{\phi}^{-1}\left(\theta_{0}\right)$ : the transition matrix of relative motion

- $\phi(\theta)$ : the Yamanaka-Ankersen fundamental matrix of relative motion

- $\boldsymbol{R}(\theta)$ : primer vector reduced matrix

- $N$ : number of velocity increments

- $d_{\theta}$ : the duration of the rendezvous, rad

- $\theta_{i}, \forall i=1, \cdots, N$ : impulses application locations, rad

- $\delta\left(\theta-\theta_{i}\right)$ : Dirac impulse at $\theta_{i}$

- $\Delta V\left(\theta_{i}\right):$ velocity increment at $\theta_{i}, \mathrm{~m} / \mathrm{s}$

- $\left\{b_{i}\right\}_{i=1, \cdots, N}$ : sequence of variables $b_{i}, \forall i=1, \cdots, N$;

- $\operatorname{sgn}(z)$ : sign of the variable $z$

- $|X|$ : the absolute value of the scalar $X$

- $\|\vec{u}\|_{p}: p$-norm of the vector $\vec{u}$

- $\|\vec{f}(t)\|_{p}=\int_{t_{0}}^{t_{f}}\|\vec{f}(t)\|_{p} d t: \mathcal{L}_{1}$ norm of the $p$-norm of the vectorial function $\vec{f}(t)$

\section{PROBLEM FORMULATION}

Let us consider a typical fixed-time minimum-fuel rendezvous situation between elliptic orbits of an active spacecraft and a passive target spacecraft. The two orbits are assumed sufficiently close to allow for the linearization of the relative equations of motion. These equations are written in a moving Local Vertical Local Horizontal (LVLH) frame, attached to the gravity center of the passive target and which rotates with its angular velocity, as illustrated in Figure 1

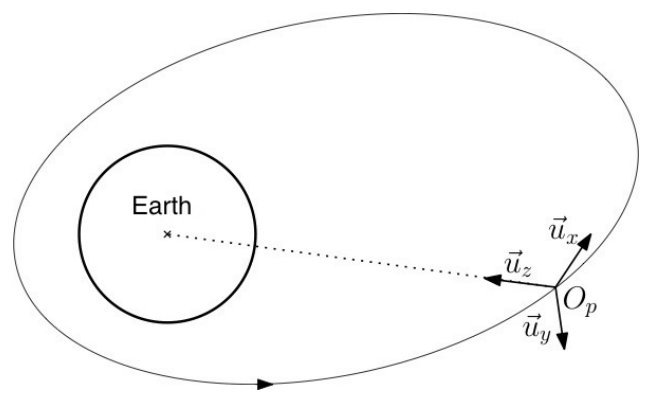

Fig. 1. Local Vertical Local Horizontal (LVLH) frame attached to the target spacecraft: the $z$-axis is the radial direction, oriented towards the center of the Earth. The $y$-axis is perpendicular to the orbital plane of the chaser, pointing opposite the orbital angular momentum. The $x$-axis completes the frame.

Under Keplerian and linearizing close-proximity assumptions, the complete rendezvous problem may be decoupled between the out-of-plane rendezvous problem and the coplanar problem. An analytical solution of the out-of-plane rendezvous problem may be found in [3] when $e=0$ while numerous studies exist for the coplanar rendezvous problem [9], [10], [1], [2], [11] and [12], [13] for more recent references. Note that when the fuel-optimal solutions are obtained independently for the coplanar case and the out-of-plane case, the complete optimal solution for the 3-dimensional, sixth-order fuel-optimal linearized rendezvous problem is simply obtained by gathering the two previous planning solutions in a single planning solution based on a 3-dimensional control vector. The Tschauner-Hempel equation for the relative out-of-plane motion is given by:

$$
\begin{aligned}
\ddot{y}(t) & =-n^{2} \frac{(1+e \cos (\theta))^{3}}{\left(1-e^{2}\right)^{3}} y(t)+\frac{F_{y}(t)}{m(t)}, \\
\dot{m}(t) & =-\alpha(t)\left\|F_{y}(t)\right\|_{p}
\end{aligned}
$$

where $n, 0 \leq e<1$ and $\theta$ are respectively the mean motion, the eccentricity and the true anomaly of the reference orbit while $m(t)$ is the vehicle mass, $\alpha(t)$ is a nonnegative known function of time and $F_{y}(t) / m(t)$ is the force per unit of mass (acceleration) applied by the thruster to the active spacecraft. As has been done in [14], time is replaced by $\theta$ as a convenient independent variable and the simplifying change of variables $\tilde{y}(\theta)=(1+e \cos (\theta)) y(\theta)$ is applied to the differential equation resulting in the simplified equation:

$$
\tilde{y}^{\prime \prime}(\theta)=-\tilde{y}(\theta)+\tilde{u}_{y}(\theta),
$$

where $(\cdot)^{\prime}=d(\cdot) / d \theta$ and $\tilde{u}_{y}(\theta)=\frac{\left(1-e^{2}\right)^{3} F_{y}(\theta)}{n^{2} m(\theta)(1+e \cos (\theta))^{3}}$. Note also that $\dot{\theta}=\frac{d \theta}{d t}=n\left(1-e^{2}\right)^{-\frac{3}{2}}(1+e \cos (\theta))^{2}$. 
Depending on the geometrical configuration of the thrust engines mounted to the body axes of the spacecraft, the fuel consumption is usually defined as the $\mathcal{L}_{1}$-norm of the $p$-norm of the acceleration ( $p=1$ for 6 ungimbaled thrusters and $p=2$ for a single gimbaled thruster). In the particular case of the out-of-plane rendezvous, the control is one-dimensional, so that the fuel consumption is in both cases expressed as:

$$
J=\left\|\frac{F_{y}(t)}{m(t)}\right\|_{1, p}=\int_{t_{0}}^{t_{f}} \frac{\left\|F_{y}(t)\right\|_{p}}{m(t)} \mathrm{d} t=\int_{t_{0}}^{t_{f}} \frac{\left|F_{y}(t)\right|}{m(t)} \mathrm{d} t .
$$

Using the independent variable $\theta$ and the simplifying change of variable defined above, the performance index becomes:

$$
\begin{aligned}
J\left(\bar{u}_{y}\right) & =n\left(1-e^{2}\right)^{-3 / 2} \int_{\theta_{0}}^{\theta_{f}}(1+e \cos (\theta))\left|\tilde{u}_{y}(\theta)\right| \mathrm{d} \theta \\
& =\int_{\theta_{0}}^{\theta_{f}}\left|\bar{u}_{y}(\theta)\right| \mathrm{d} \theta
\end{aligned}
$$

where $\theta_{0}$ and $\theta_{f}$ respectively denote the fixed initial and final values of the true anomaly during the rendezvous and where the following change of variables $\tilde{u}_{y}(\theta)=\frac{\left(1-e^{2}\right)^{3 / 2}}{n(1+e \cos (\theta))} \bar{u}_{y}(\theta)$ is used. In the LVLH frame, the state vector of the active spacecraft characterizing the relative out-of-plane motion is composed of its relative position and velocity to the target: $\overrightarrow{\widetilde{X}}(\theta)=\left[\begin{array}{lll}\tilde{y}(\theta) & \tilde{y}^{\prime}(\theta)\end{array}\right]^{T}$. The fixed-time out-of-plane fueloptimal rendezvous problem is then defined as the following optimal control problem:

$$
\begin{aligned}
& \min _{u_{y} \in \mathcal{L}_{1}\left(\left[\theta_{0}, \theta_{f}\right], \mathbb{R}\right)} J\left(\bar{u}_{y}\right) \\
& \text { s.t. } \\
& \qquad \overrightarrow{\widetilde{X}}^{\prime}(\theta)=\left[\begin{array}{cc}
0 & 1 \\
-1 & 0
\end{array}\right] \overrightarrow{\widetilde{X}}(\theta)+\left[\begin{array}{c}
0 \\
\frac{\left(1-e^{2}\right)^{3 / 2}}{n(1+e \cos (\theta))}
\end{array}\right] \bar{u}_{y}(\theta), \\
& \quad \overrightarrow{\widetilde{X}}\left(\theta_{0}\right)=\overrightarrow{\widetilde{X}}_{0}, \overrightarrow{\widetilde{X}}\left(\theta_{f}\right)=\overrightarrow{\widetilde{X}}_{f} \text { fixed, } \theta_{0}, \theta_{f} \text { fixed, }
\end{aligned}
$$

where $\mathcal{L}_{1}\left(\left[\theta_{0}, \theta_{f}\right], \mathbb{R}\right)$ is the normed linear space of Lebesgue integrable functions from $\left[\theta_{0}, \theta_{f}\right]$ to $\mathbb{R}$ equipped with the norm defined above. Note that the final value $\vec{X}_{f}$ for the state vector may be different from 0 according to the terminology used by T. Carter for the rendezvous problem [1]. L.W. Neustadt has proved in [6] that the solution of (5] may be arbitrarily approximated by a finite linear combination of $N$ $\delta(\cdot)$ functions: $\bar{u}_{\epsilon}(\theta) \rightarrow \sum_{j=1}^{N} \Delta V\left(\theta_{j}\right) \delta\left(\theta_{j}-\theta\right), \epsilon \rightarrow 0$, where $\Delta V\left(\theta_{i}\right)$ is the velocity increment applied at $\theta_{i}$. Only an upper bound ( 2 in the out-of-plane case) on the optimal number $N$ of velocity increments is known [6]. Using the fundamental matrix $\phi(\theta)$ of the differential equation in (5) defined as:

$$
\phi(\theta)=\left[\begin{array}{cc}
\cos (\theta) & \sin (\theta) \\
-\sin (\theta) & \cos (\theta)
\end{array}\right]
$$

we get the solution of the equation in 5 for $N$ impulses as:

$$
\begin{aligned}
\overrightarrow{\widetilde{X}}_{f}= & \phi\left(\theta_{f}\right) \boldsymbol{\phi}^{-1}\left(\theta_{0}\right) \overrightarrow{\widetilde{X}}_{0} \\
& +\sum_{i=1}^{N} \phi\left(\theta_{f}\right) \boldsymbol{\phi}^{-1}\left(\theta_{i}\right)\left[\begin{array}{c}
0 \\
\frac{\left(1-e^{2}\right)^{3 / 2}}{n(1+e \cos (\theta))}
\end{array}\right] \Delta V\left(\theta_{i}\right)
\end{aligned}
$$

Defining the following notations,

$$
\begin{gathered}
\boldsymbol{R}(\theta)=\phi(\theta)^{-1}\left[\begin{array}{l}
0 \\
1
\end{array}\right]=\left[\begin{array}{c}
-\sin (\theta) \\
\cos (\theta)
\end{array}\right] \\
r(\theta)=1+e \cos (\theta) \\
\vec{z}_{f}=\left[\begin{array}{c}
z_{f_{1}} \\
z_{f_{2}}
\end{array}\right]=n\left(1-e^{2}\right)^{-\frac{3}{2}}\left(\phi^{-1}\left(\theta_{f}\right) \overrightarrow{\widetilde{X}}_{f}-\phi^{-1}\left(\theta_{0}\right) \overrightarrow{\widetilde{X}}_{0}\right) \neq 0,
\end{gathered}
$$

the initial optimal control problem (5) amounts to find the sequences of optimal impulse locations $\left\{\theta_{i}\right\}_{i=1, \cdots, N}$ and optimal velocity increments $\left\{\Delta V\left(\theta_{i}\right)\right\}_{i=1, \cdots, N}$ solution of the following optimization problem:

$$
\min _{\theta_{i}, \Delta V\left(\theta_{i}\right)} J=\sum_{i=1}^{N}\left|\Delta V\left(\theta_{i}\right)\right| \text { s.t. } \vec{z}_{f}=\sum_{i=1}^{N} \frac{\boldsymbol{R}\left(\theta_{i}\right)}{r\left(\theta_{i}\right)} \Delta V\left(\theta_{i}\right) .
$$

The optimization decision variables are the sequence of thrust locations $\left\{\theta_{i}\right\}_{i=1, \cdots, N}$ and the sequence of thrusts $\left\{\Delta V\left(\theta_{i}\right)\right\}_{i=1, \cdots, N}$.

Due to the lack of a priori information about the optimal number of impulses to be considered, problem (10) is very hard to solve from both theoretical and numerical points of view. Therefore, the associated fixed-time minimum-fuel rendezvous problem for a fixed number $N$ of impulses has been considered in the literature mainly via geometric methods near circular [9], [10], [11] or elliptic [2] orbits. These results are mainly based on the derivation of optimality conditions for the problem (10) when $N$ is fixed a priori. These optimality conditions are now recalled in the framework of [2].

\section{OPTIMALITY CONDITIONS}

Applying the maximum Principle to the original optimal control problem (5) for a fixed number of impulses and under the impulsive approximation as described in Lawden [15], or a Lagrange multiplier rule for the equivalent Problem 10 as in [2], one can derive necessary conditions of optimality in terms of the co-state vector associated with the relative velocity and referred to as the primer vector (see conditions (11) to (14) in Theorem 1). These conditions are also sufficient in the case of linear relative motion when strengthening them by adding the semi-infinite constraint (15) that should be fulfilled on the continuum $\left[\theta_{0}, \theta_{f}\right][16]$. These results are summarized using the formalism of T. Carter in the following theorem.

Theorem 1 ([15], [6]): $\left(\theta_{1}, \ldots, \theta_{N}, \Delta V\left(\theta_{1}\right), \ldots, \Delta V\left(\theta_{N}\right)\right)$ is an optimal solution of problem (10) if and only if there exists a non-zero vector $\vec{\lambda} \in \mathbb{R}^{m}, m=\operatorname{dim}\left(\vec{z}_{f}\right)$ that verifies the necessary and sufficient conditions: for all $i=1, \cdots, N$,

$$
\begin{array}{r}
\Delta V\left(\theta_{i}\right)=-p\left(\theta_{i}\right)\left|\Delta V\left(\theta_{i}\right)\right|, \\
\left|\Delta V\left(\theta_{i}\right)\right|=0 \text { or }\left|p\left(\theta_{i}\right)\right|=1, \\
\left|\Delta V\left(\theta_{i}\right)\right|=0 \text { or } \theta_{i} \in\left\{\theta_{0}, \theta_{f}\right\} \text { or } \frac{d|p|^{2}}{d \theta}\left(\theta_{i}\right)=0, \\
\sum_{i=1}^{N} \frac{\boldsymbol{R}\left(\theta_{i}\right)}{r\left(\theta_{i}\right)} p\left(\theta_{i}\right)\left|\Delta V\left(\theta_{i}\right)\right|=-\vec{z}_{f}, \\
|p(\theta)| \leq 1, \forall \theta \in\left[\theta_{0}, \theta_{f}\right],
\end{array}
$$


where $p(\theta)$ is the so-called primer vector [17] and is defined, in the out-of-plane rendezvous problem as:

$$
p(\theta)=\frac{\boldsymbol{R}(\theta)^{T} \vec{\lambda}}{r(\theta)}=\frac{-\lambda_{1} \sin (\theta)+\lambda_{2} \cos (\theta)}{1+e \cos (\theta)} .
$$

These results date back to the seminal work of D.F. Lawden [15] in the early sixties, where the so-called primer vector theory has been quoted for the first time and proved rigorously later by L. Neustadt in [6]. Roughly speaking, when applying the Pontryagin principle maximum, the primer vector is defined as the part of the costate vector associated to the relative velocity and indicates the optimal thrusting directions in the optimality conditions (see the reference [18, Chapter 2] for more details on primer vector theory).

Obviously a primer vector candidate is completely defined by the choice of the Lagrange multipliers $\lambda_{1}, \lambda_{2}$.

\section{PRIMER VECTOR CANDIDATE DYNAMICS}

In this section, the particular properties of the primer vector are analyzed such that these characteristics may be used for the derivation of the optimal solutions.

By $(16), p(\theta)$ is obviously a $2 \pi$-periodic function. It is a harmonic oscillator divided by the positive function $r(\theta)=$ $1+e \cos (\theta)$. As a result, its sign changes every $\pi$. Its derivative may be calculated as follows:

$$
\frac{\mathrm{d} p}{\mathrm{~d} \theta}(\theta)=-\frac{\lambda_{1}(e+\cos (\theta))+\lambda_{2} \sin (\theta)}{(1+e \cos (\theta))^{2}} .
$$

As $0 \leq e<1$, it is easy to deduce that $p(\theta)$ reaches two global extrema of opposite sign at $\theta_{e_{1}}$ and $\theta_{e_{2}}$ modulo $2 \pi$.

\section{A. Lagrange multipliers as functions of an extremum}

If $p(\theta)$ has an extremum $p\left(\theta_{e}\right)$ at $\theta_{e}$ then it follows from (16) and (17) that:

$$
\lambda_{1}=-p\left(\theta_{e}\right) \sin \left(\theta_{e}\right), \quad \lambda_{2}=p\left(\theta_{e}\right)\left(e+\cos \left(\theta_{e}\right)\right) .
$$

A primer vector candidate can thus be rewritten as follows:

$$
p(\theta)=p\left(\theta_{e}\right) \frac{\cos \left(\theta-\theta_{e}\right)+e \cos (\theta)}{1+e \cos (\theta)} .
$$

\section{B. Extremum ratio}

From 18 and remembering that $p\left(\theta_{e_{1}}\right)=-p\left(\theta_{e_{2}}\right)$, it comes that:

$$
\begin{aligned}
& \frac{\left|p\left(\theta_{e_{2}}\right)\right|}{\left|p\left(\theta_{e_{1}}\right)\right|} \sin \left(\theta_{e_{2}}\right)=-\sin \left(\theta_{e_{1}}\right), \\
& \frac{p\left(\theta_{e_{2}}\right) \mid}{\left|p\left(\theta_{e_{1}}\right)\right|}\left(e+\cos \left(\theta_{e_{2}}\right)\right)=-\left(e+\cos \left(\theta_{e_{1}}\right)\right) .
\end{aligned}
$$

By denoting $X=\frac{\left|p\left(\theta_{e_{2}}\right)\right|}{\left|p\left(\theta_{e_{1}}\right)\right|}$ and combining equations in 20p, one can get a second order polynomial equation where the ratio of the absolute values is a solution to:

$$
X^{2}-\frac{2 e\left(e+\cos \left(\theta_{e_{1}}\right)\right)}{1-e^{2}} X-\frac{1+2 e \cos \left(\theta_{e_{1}}\right)+e^{2}}{1-e^{2}}=0 .
$$

This polynomial has two real roots $\left\{-1 ; \frac{1+2 e \cos \left(\theta_{e_{1}}\right)+e^{2}}{1-e^{2}}\right\}$ and only the second one is positive (corresponding to the ratio of absolute values), so that:

$$
X=\frac{1+2 e \cos \left(\theta_{e_{1}}\right)+e^{2}}{1-e^{2}}
$$

and

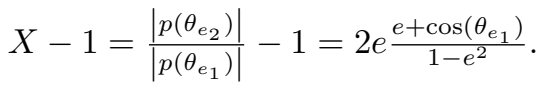

Note that $\left|p\left(\theta_{e_{1}}\right)\right| \neq 0$ otherwise $p(\theta) \equiv 0$ by (18). Suppose now that the maximum norm extremum is given by $\theta_{e_{2}}$, i.e. $\frac{\left|p\left(\theta_{e_{2}}\right)\right|}{\left|p\left(\theta_{e_{1}}\right)\right|} \geq 1$, then $\theta_{e_{1}}$ is the minimum norm extremum and it is deduced from (22) that

$$
\frac{\left|p\left(\theta_{e_{2}}\right)\right|}{\left|p\left(\theta_{e_{1}}\right)\right|}-1=2 e \frac{e+\cos \left(\theta_{e_{1}}\right)}{1-e^{2}} \geq 0 .
$$

Therefore, $\cos \left(\theta_{e_{1}}\right) \geq-e$ for the minimum norm extremum and the maximum norm extremum is such that $\cos \left(\theta_{e}\right) \leq-e$ which is obtained the same way by assuming that $\theta_{e_{2}}$ is the minimum norm extremum. When $\left|p\left(\theta_{e_{1}}\right)\right|=1$ it comes that:

$$
\left|p\left(\theta_{e_{2}}\right)\right|=\frac{1+2 e \cos \left(\theta_{e_{1}}\right)+e^{2}}{1-e^{2}} .
$$

Thus $\left|p\left(\theta_{e_{2}}\right)\right|>1$ if and only if $\cos \left(\theta_{e_{1}}\right)>-e$.

\section{Extremum as a function of the Lagrange multipliers}

Using 18 , for a given $\vec{\lambda} \in \mathbb{R}^{2}$ such that $\lambda_{2} \neq 0$, the anomalies $\theta_{e_{1}}$ and $\theta_{e_{2}}$ for which the primer vector reaches its two extrema are given by the equation:

$$
\frac{\sin \left(\theta_{e}\right)}{e+\cos \left(\theta_{e}\right)}=-\frac{\lambda_{1}}{\lambda_{2}} \text {. }
$$

By defining $Y=\cos \left(\theta_{e}\right)$ and $Q=\frac{\lambda_{1}}{\lambda_{2}}$, it follows after taking the square of $(26)$ that:

$$
\left(1+Q^{2}\right) Y^{2}+2 e Q^{2} Y+e^{2} Q^{2}-1=0 .
$$

The roots of 27 are $\frac{ \pm \sqrt{1+Q^{2}\left(1-e^{2}\right)}-e Q^{2}}{1+Q^{2}}$. So that:

$$
\begin{aligned}
& \cos \left(\theta_{e}\right)=\frac{ \pm \sqrt{1+Q^{2}\left(1-e^{2}\right)}-e Q^{2}}{1+Q^{2}} \\
& \sin \left(\theta_{e}\right)=-Q \frac{ \pm \sqrt{1+Q^{2}\left(1-e^{2}\right)}+e}{1+Q^{2}} .
\end{aligned}
$$

Thus, keeping in mind the restrictions on the maximum and minimum norm extremum, the maximum and minimum norm value of the primer vector can be expressed in terms of $\vec{\lambda}$. Inserting the expressions of $\cos \left(\theta_{e}\right)$ and $\sin \left(\theta_{e}\right)$ of (28) in (16), the following expression is obtained after noting that $\lambda_{1}=Q \lambda_{2}$ :

$$
p\left(\theta_{e}\right)=\frac{ \pm \lambda_{2}\left(1+Q^{2}\right) \sqrt{1+Q^{2}\left(1-e^{2}\right)}}{1+\left(1-e^{2}\right) Q^{2} \pm e \sqrt{1+Q^{2}\left(1-e^{2}\right)}} .
$$

Noting that (by completing the square):

$$
\begin{aligned}
& 1+\left(1-e^{2}\right) Q^{2}+e \sqrt{1+Q^{2}\left(1-e^{2}\right)} \\
& =\sqrt{1+Q^{2}\left(1-e^{2}\right)}\left(\sqrt{1+Q^{2}\left(1-e^{2}\right)}+e\right) \\
& 1+\left(1-e^{2}\right) Q^{2}-e \sqrt{1+Q^{2}\left(1-e^{2}\right)} \\
& =\sqrt{1+Q^{2}\left(1-e^{2}\right)}\left(\sqrt{1+Q^{2}\left(1-e^{2}\right)}-e\right)
\end{aligned}
$$

and taking the absolute value of 29], the following expressions are obtained:

$$
\begin{aligned}
& \max _{\theta \in \mathbb{R}}|p(\theta)|=\frac{\left|\lambda_{2}\right|\left(1+Q^{2}\right)}{\sqrt{1+Q^{2}\left(1-e^{2}\right)}-e}, \\
& \min _{\theta \in \mathbb{R}}|p(\theta)|=\frac{\left|\lambda_{2}\right|\left(1+Q^{2}\right)}{\sqrt{1+Q^{2}\left(1-e^{2}\right)}+e} .
\end{aligned}
$$




\section{Minimum-FUEL OOP OPTIMAL SOLUTIONS}

When looking for the complete solution of the minimumfuel OOP problem, the primary difficulty lies in the lack of knowledge of the exact basic structure of the optimal trajectory. Indeed, the optimal number and optimal locations of impulses are not a priori given but are instead optimization variables of the optimization process. The method used to derive the analytical solution of the problem mainly consists in exploiting the different features of the optimal primer vector exhibited in the previous section and in discussing all its different possible configurations for optimal plan of maneuvers, depending upon the duration of the rendezvous $d_{\theta}=\theta_{f}-\theta_{0}$, the eccentricity $e$ of the target orbit, the initial and final anomalies $\theta_{0}$ and $\theta_{f}$ and upon the vector $\vec{z}_{f}$. Bearing in mind that the upper bound on the optimal number of impulses given by Neustadt is 2 for the minimum-fuel OOP problem and that an optimal impulse may be located either at the interior or at the boundary of the interval $\left[\theta_{0}, \theta_{f}\right]$, it is then possible to enumerate all the possible optimal solutions and to derive the conditions linked to their occurence. These conditions are expressed in terms of the parameters $d_{\theta}, e$, $\theta_{0}, \theta_{f}$ and $\vec{z}_{f}$. A preliminary analysis on the duration of the rendezvous provides a structure to the discussion of the different cases.

- When the duration of the rendezvous is longer than a period (i.e. when $d_{\theta} \geq 2 \pi$ ), the primer vector $p(\theta)$ will reach its local extrema on $\left[\theta_{0}, \theta_{f}\right]$. Therefore, only two possibilities may occur for $p(\theta)$ to be optimal since it must verify (15): one extremum has a unit norm and the other has a norm strictly less than 1 (one impulse per $2 \pi$ ), or both extrema of $p(\theta)$ have a unit norm (two impulses, at most, per $2 \pi$ ).

- When the duration of the rendezvous is shorter than a period (i.e. when $d_{\theta}<2 \pi$ ), this dependency may be quite complicated since an extremum could be at an end point, as illustrated by the next subsections.

Next, the different cases are discussed in the clearest way possible. For each type of optimal solution, the associated conditions involving $e, \theta_{0}, \theta_{f}$ and $\vec{z}_{f}$ are given. The optimal Lagrange multipliers and related primer vector are then presented. A summary of all the optimal solutions is then given. Let us first define some notations needed in the sequel:

$$
\begin{aligned}
& \varepsilon_{1}=\operatorname{sgn}\left(z_{f_{1}}\right), \varepsilon_{2}=\operatorname{sgn}\left(z_{f_{2}}\right), \\
& \varepsilon_{0}=\operatorname{sgn}\left(\cos \left(\theta_{0}\right) z_{f_{1}}+\sin \left(\theta_{0}\right) z_{f_{2}}\right), \\
& \varepsilon_{f}=\operatorname{sgn}\left(\cos \left(\theta_{f}\right) z_{f_{1}}+\sin \left(\theta_{f}\right) z_{f_{2}}\right) .
\end{aligned}
$$

\section{A. Two interior impulses solution}

In this section, the case of optimal solutions with two interior impulses per period is investigated. Independently of the duration of the rendezvous, the optimal primer vector will reach its two extrema, and both extrema have a unit norm.

$$
\theta_{ \pm}=\min \left\{\theta \geq \theta_{0} \text { s.t. }\left\{\begin{array}{l}
\cos (\theta)=-e \\
\sin (\theta)= \pm \sqrt{1-e^{2}}
\end{array}\right\}\right.
$$

The notation $\theta_{ \pm}$defines two different locations $\theta_{+}$and $\theta_{-}$ belonging to the interval $\left[\theta_{0}, \theta_{0}+2 \pi\right)$. They are defined by an identical cosine and by a positive or a negative sine respectively. Note that when $d_{\theta}<\pi$, due to (32), the condition $\left(\theta_{-}, \theta_{+}\right) \in\left[\theta_{0}, \theta_{f}\right]^{2}$ is equivalent to:

$$
\sin \left(\theta_{0}\right) \geq \sqrt{1-e^{2}} \text { and } \sin \left(\theta_{f}\right) \leq-\sqrt{1-e^{2}} .
$$

When $\pi \leq d_{\theta}<2 \pi$, the condition $\left(\theta_{-}, \theta_{+}\right) \in\left[\theta_{0}, \theta_{f}\right]^{2}$ is equivalent to the three possible configurations expressed by:

$$
\begin{aligned}
& \sin \left(\theta_{0}\right) \geq \sqrt{1-e^{2}} \\
& \text { or }\left\{\begin{array}{l}
\sin \left(\theta_{0}\right) \leq-\sqrt{1-e^{2}} \\
\text { and } \sin \left(\theta_{f}\right) \leq-\sqrt{1-e^{2}}
\end{array}\right. \\
& \text { or }\left\{\begin{array}{l}
\left|\sin \left(\theta_{0}\right)\right|<\sqrt{1-e^{2}} \\
\text { and }\left(e+\cos \left(\theta_{0}\right)\right)\left(e+\cos \left(\theta_{f}\right)\right)>0 .
\end{array}\right.
\end{aligned}
$$

Proposition 1: An optimal solution for the linearized impulsive out-of-plane rendezvous problem is a 2-impulse trajectory defined by the optimal locations $\theta_{ \pm}$and the optimal directions of thrust given by:

$$
\Delta V\left(\theta_{ \pm}\right)=\frac{\sqrt{1-e^{2}}}{2 e}\left(\mp e z_{f_{1}}-\sqrt{1-e^{2}} z_{f_{2}}\right),
$$

if the following conditions are verified:

$$
e\left|\vec{z}_{f}\right|>\left|z_{f_{2}}\right| \text { and } d_{\theta} \geq 2 \pi
$$

or

$$
\begin{aligned}
& e\left|\vec{z}_{f}\right|>\left|z_{f_{2}}\right| \text { and } d_{\theta}<\pi \text { and } \\
& \sin \left(\theta_{0}\right) \geq \sqrt{1-e^{2}} \text { and } \sin \left(\theta_{f}\right) \leq-\sqrt{1-e^{2}}
\end{aligned}
$$

or

$$
e\left|\vec{z}_{f}\right|>\left|z_{f_{2}}\right| \text { and } \pi \leq d_{\theta}<2 \pi \text { and } 34
$$

Finally, the optimal Lagrange multipliers and the optimal primer vector are:

$$
\lambda_{1}=-\varepsilon_{1} \sqrt{1-e^{2}}, \quad \lambda_{2}=0, \quad p(\theta)=\frac{\varepsilon_{1} \sqrt{1-e^{2}} \sin (\theta)}{1+e \cos (\theta)} .
$$

REMARK 1: As indicated in the proof of Proposition 1 given in the Appendix, when $d_{\theta} \geq 2 \pi$, the optimal solution of the planning may be chosen to be spread over $N_{-}+N_{+}$ impulses verifying (88) and 89, depending on the duration of the rendezvous and operational constraints while preserving the optimal consumption.

\section{B. One interior impulse solutions}

Consider the case of optimal solutions with only one interior impulse and no boundary impulse. In this case, two geometrical configurations of the primer vector may occur. When the rendezvous lasts more than a period $\left(d_{\theta} \geq 2 \pi\right)$, the associated optimal primer vector necessarily reaches one extremum of unit norm while the other has a norm strictly less than 1 . When the rendezvous lasts less than a period $\left(d_{\theta}<2 \pi\right)$, there is no condition on the other extremum norm and additional conditions will be needed to ensure $|p(\theta)| \leq 1, \theta \in\left[\theta_{0}, \theta_{f}\right]$.

Proposition 2: Provided that $\cos \left(\theta_{0}\right) z_{f_{1}}+\sin \left(\theta_{0}\right) z_{f_{2}} \neq 0$ and $\cos \left(\theta_{f}\right) z_{f_{1}}+\sin \left(\theta_{f}\right) z_{f_{2}} \neq 0$, an optimal solution for the 
linearized impulsive out-of-plane rendezvous problem is a 1impulse trajectory defined by the optimal locations $\theta_{i b}$ :

$$
\cos \left(\theta_{i b}\right)=-\varepsilon_{b} \frac{z_{f_{2}}}{\left|\vec{z}_{f}\right|}, \sin \left(\theta_{i b}\right)=\varepsilon_{b} \frac{z_{f_{1}}}{\left|\vec{z}_{f}\right|},
$$

and optimal thrusts are defined by:

$$
\Delta V\left(\theta_{i b}\right)=-\varepsilon_{b}\left|\vec{z}_{f}\right|+e z_{f_{2}},
$$

with index $b=0$, if the following conditions are verified:

$$
d_{\theta}<2 \pi \text { and } e\left|\vec{z}_{f}\right| \leq \varepsilon_{0} z_{f_{2}} \text { and } \varepsilon_{f}=-\varepsilon_{0}
$$

or

$$
\begin{gathered}
d_{\theta}<2 \pi \text { and } e\left|\vec{z}_{f}\right|>\varepsilon_{0} z_{f_{2}} \text { and } \varepsilon_{f}=-\varepsilon_{0} \\
\text { and } \\
\left\{\begin{array}{rr}
\left|\vec{z}_{f}\right|+\left(2 e\left|\vec{z}_{f}\right|-\varepsilon_{0} z_{f_{2}}\right) \cos \left(\theta_{0}\right)+\varepsilon_{0} z_{f_{1}} \sin \left(\theta_{0}\right) & >0 \\
\left|\vec{z}_{f}\right|+\left(2 e\left|\vec{z}_{f}\right|-\varepsilon_{0} z_{f_{2}}\right) \cos \left(\theta_{f}\right)+\varepsilon_{0} z_{f_{1}} \sin \left(\theta_{f}\right) & >0 \\
\varepsilon_{0}\left(e+\cos \left(\theta_{0}\right)\right) z_{f_{1}}+\left(\varepsilon_{0} z_{f_{2}}-e\left|\vec{z}_{f}\right|\right) \sin \left(\theta_{0}\right) & >0 \\
\varepsilon_{0}\left(e+\cos \left(\theta_{f}\right)\right) z_{f_{1}}+\left(\varepsilon_{0} z_{f_{2}}-e\left|\vec{z}_{f}\right|\right) \sin \left(\theta_{f}\right) & <0
\end{array}\right.
\end{gathered}
$$

with index $b=2$, if the following conditions are verified:

$$
d_{\theta} \geq 2 \pi \text { and }\left|z_{f_{2}}\right|>e\left|\vec{z}_{f}\right|
$$

or

$$
\pi \leq d_{\theta}<2 \pi \text { and } e\left|\vec{z}_{f}\right| \leq\left|z_{f_{2}}\right| \text { and } \varepsilon_{f}=\varepsilon_{0}
$$

or

$$
\begin{gathered}
\pi \leq d_{\theta}<2 \pi \text { and } e\left|\vec{z}_{f}\right|>\left|z_{f_{2}}\right| \text { and } \varepsilon_{f}=\varepsilon_{0} \\
\text { and } \\
\left\{\begin{array}{rr}
\left|\vec{z}_{f}\right|+\left(2 e\left|\vec{z}_{f}\right|-\varepsilon_{2} z_{f_{2}}\right) \cos \left(\theta_{0}\right)+\varepsilon_{2} z_{f_{1}} \sin \left(\theta_{0}\right) & >0 \\
\left|\vec{z}_{f}\right|+\left(2 e\left|\vec{z}_{f}\right|-\varepsilon_{2} z_{f_{2}}\right) \cos \left(\theta_{f}\right)+\varepsilon_{2} z_{f_{1}} \sin \left(\theta_{f}\right) & >0 \\
\varepsilon_{2} z_{f_{1}}\left(e+\cos \left(\theta_{0}\right)\right)-\left(e\left|\vec{z}_{f}\right|-\varepsilon_{2} z_{f_{2}}\right) \sin \left(\theta_{0}\right) & >0 \\
-\varepsilon_{2} z_{f_{1}}\left(e+\cos \left(\theta_{f}\right)\right)+\left(e\left|\vec{z}_{f}\right|-\varepsilon_{2} z_{f_{2}}\right) \sin \left(\theta_{f}\right) & >0 .
\end{array}\right.
\end{gathered}
$$

Finally, the optimal Lagrange multipliers and the optimal primer vector are

$$
\begin{aligned}
& \lambda_{1}=-\frac{z_{f_{1}}}{\left|\vec{z}_{f}\right|}, \lambda_{2}=\varepsilon_{b} e-\frac{z_{f_{2}}}{\left|\vec{z}_{f}\right|}, \\
& p(\theta)=\frac{z_{f_{1}} \sin (\theta)+\left(\varepsilon_{b} e\left|\vec{z}_{f}\right|-z_{f_{2}}\right) \cos (\theta)}{(1+e \cos (\theta))\left|\vec{z}_{f}\right|} .
\end{aligned}
$$

REMARK 2: For the case $d_{\theta} \geq 2 \pi$, a remark similar to the Remark 1 may be stated. The first true anomaly $\theta_{i 2}$ in $\left[\theta_{0}, \theta_{f}\right]$ for which the primer vector reaches its extremum of unit norm, is defined by:

$$
\theta_{i 2}=\min \left\{\theta \in\left[\theta_{0}, \theta_{f}\right]:|p(\theta)|=1 \text { and } \frac{\mathrm{d} p}{\mathrm{~d} \theta}(\theta)=0\right\}
$$

From the extremum ratio (25) and (40), it comes that: $\cos \left(\theta_{i 2}\right)<-e$, and: $\left|\vec{z}_{f}\right|-e\left|z_{f_{2}}\right|>0$. In this particular case, the optimal solution may exhibit, at most, $N^{*}$ impulses, located at:

$$
\theta_{i}=\theta_{i 2}+2(i-1) \pi, i=1, \cdots, N^{*},
$$

where: $N^{*}=\max \left\{i \in \mathbb{N}^{*}: \theta_{i 2}+2(i-1) \pi \leq \theta_{f}\right\}$. These $N^{*}$ optimal impulses may be chosen arbitrarily as long as the minimum fuel consumption is preserved i.e.:

$$
\sum_{i=1}^{N^{*}}\left|\Delta V\left(\theta_{i}\right)\right|=\left|\vec{z}_{f}\right|-e\left|z_{f_{2}}\right| .
$$

Depending on the operational constraints, the optimal solution may be reduced to a single impulse at $\theta_{i 2}: \Delta V\left(\theta_{i 2}\right)=$ $-\varepsilon_{2}\left(\left|\vec{z}_{f}\right|-e\left|z_{f_{2}}\right|\right)$ as stated in Proposition 2, or spread over $N^{*}$ impulses $\Delta V\left(\theta_{i}\right)$ located at $\theta_{i}$ given by 48 and under the constraint 49.

A particular case of Proposition 2 is when the primer vector has a unit norm extremum at $\theta_{0}$ (respectively at $\theta_{f}$ ). The results in this case are summarized below.

Corollary 1: When $\cos \left(\theta_{0}\right) z_{f_{1}}+\sin \left(\theta_{0}\right) z_{f_{2}}=0$ (resp., when $\cos \left(\theta_{f}\right) z_{f_{1}}+\sin \left(\theta_{f}\right) z_{f_{2}}=0$ ), the optimal solution comes down to a one impulse boundary solution:

$$
\Delta V\left(\theta_{b}\right)=\left(-\sin \left(\theta_{b}\right) z_{f_{1}}+\cos \left(\theta_{b}\right) z_{f_{2}}\right)\left(1+e \cos \left(\theta_{b}\right)\right),
$$

where $b=0$ (resp. $b=f$ ), for which there may exist an infinite number of primer vectors. Without loss of generality, the primer vector may be chosen as in (47) by replacing $\varepsilon_{b}$ by $\widetilde{\varepsilon}_{b}=-\operatorname{sgn}\left(-\sin \left(\theta_{b}\right) z_{f_{1}}+\cos \left(\theta_{b}\right) z_{f_{2}}\right)$ with $b=0$ (resp. $b=f)$.

An example of the particular case ruled by Corollary 1 may be obtained with the following parameters: $e=0.8$, $\vec{z}_{f}^{T}=\left[\begin{array}{ll}-0.7297 & 2.0305\end{array}\right], \theta_{0}=3.4866, \theta_{f}=5.9998$ that have been chosen to meet the conditions above. In this case, the optimal Lagrange multipliers are given by: $\lambda_{1}=0.3382$, $\lambda_{2}=-0.1411$. The optimal primer vector, as a function of $\theta$, (dashed line) is depicted at Figure 2 alongside samples of non optimal primer vectors (solid lines) built from $\lambda_{1}$ and $\lambda_{2}$ verifying the optimality condition $(11)$ :

$$
-\lambda_{1} \sin \left(\theta_{0}\right)+\lambda_{2} \cos \left(\theta_{0}\right)=\widetilde{\varepsilon}_{0}\left(1+e \cos \left(\theta_{0}\right)\right)
$$

but not the optimality condition (15). Corollary 1 states that in

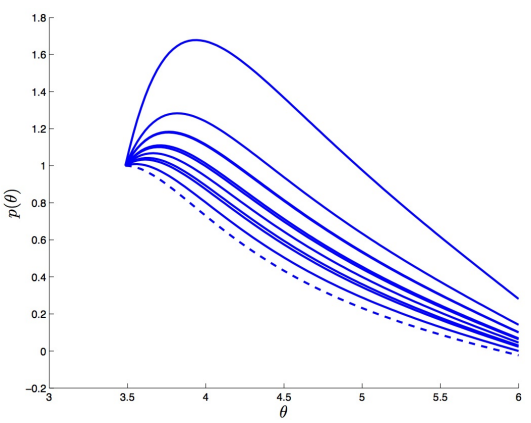

Fig. 2. Optimal (dashed line) and non optimal (solid lines) primer vector $p(\theta)$ in a particular case of Corollary 1

some cases, even if there is a unique optimal one-impulse solution to the OOP rendezvous problem, the optimal Lagrange multipliers associated to this solution are not unique and so is the possible primer vector that is not unique either. Even in this case, there will always exist one optimal primer vector (dashed curve) complying to all the optimality conditions while the other primer vectors (solid curves) will violate the optimality condition $|p(\theta)| \leq 1, \forall \theta \in\left[\theta_{0}, \theta_{f}\right]$.

\section{Initial (or final) and one interior impulses}

The focus is now on optimal solutions with one interior impulse and at least one boundary (initial or final) impulse. 
In that case, one extremum of the primer vector has a norm equal to 1 while the other has a norm greater than 1 .

Let $\theta_{i_{0}}$ denote the extremum anomaly on $\left[\theta_{0}, \theta_{f}\right]$ and $\varepsilon=$ $\frac{\Delta V\left(\theta_{i_{0}}\right)}{\left|\Delta V\left(\theta_{i_{0}}\right)\right|}$ the direction of the impulse located at $\theta_{i_{0}}$. Note that if the primer vector norm reaches 1 at any other anomaly on $\left[\theta_{0}, \theta_{f}\right]$, it can only be at $\theta_{0}$ or $\theta_{f}$ and the directions of the optimal thrusts at these locations are in the opposite direction to the interior impulse:

$$
\frac{\Delta V\left(\theta_{0}\right)}{\left|\Delta V\left(\theta_{0}\right)\right|}=\frac{\Delta V\left(\theta_{f}\right)}{\left|\Delta V\left(\theta_{f}\right)\right|}=-\varepsilon .
$$

Combining the optimality conditions (11), (12) and (13), the Lagrange multipliers are uniquely defined by:

$$
\lambda_{1}=\varepsilon \sin \left(\theta_{i_{0}}\right), \lambda_{2}=-\varepsilon\left(e+\cos \left(\theta_{i_{0}}\right)\right),
$$

and the primer vector is then given by:

$$
p(\theta)=-\varepsilon \frac{\cos \left(\theta-\theta_{i_{0}}\right)+e \cos (\theta)}{1+e \cos (\theta)} .
$$

Now, it is needed to determine the location of the interior impulse. By the primer vector definition (52), having an impulse at $\theta_{0}$ leads to:

$$
1+2 e \cos \left(\theta_{0}\right)+\cos \left(\theta_{i_{0}}-\theta_{0}\right)=0 .
$$

This equation has a solution $\theta_{i_{0}}$ on $\left.] \theta_{0}, \theta_{0}+2 \pi\right]$ if and only if $\cos \left(\theta_{0}\right) \leq 0$. If this condition is satisfied, the solutions are:

$$
\begin{aligned}
& \theta_{0}^{+}=\theta_{0}+\arccos \left(-1-2 e \cos \left(\theta_{0}\right)\right), \\
& \hat{\theta}_{0}^{+}=\theta_{0}+2 \pi-\arccos \left(-1-2 e \cos \left(\theta_{0}\right)\right),
\end{aligned}
$$

Note that if $\theta_{f}-\theta_{0}<\pi$, then (53) has a unique solution on $\left[\theta_{0}, \theta_{0}+\pi\right]$, and has a solution on $\left[\theta_{0}, \theta_{f}\right]$ if and only if condition $1+2 e \cos \left(\theta_{0}\right)+\cos \left(\theta_{f}-\theta_{0}\right) \leq 0$ is satisfied. Otherwise, when $\theta_{f}-\theta_{0} \geq \pi$, then Equation (53) has a unique solution on $\left[\theta_{0}, \theta_{f}\right]$ if and only if:

$$
1+2 e \cos \left(\theta_{0}\right)+\cos \left(\theta_{f}-\theta_{0}\right)<0
$$

and two solutions if condition (55) does not hold.

Similarly, having an interior impulse at $\theta_{i_{0}}$ and an impulse at $\theta_{f}$ leads to:

$$
1+2 e \cos \left(\theta_{f}\right)+\cos \left(\theta_{i_{0}}-\theta_{f}\right)=0 .
$$

This equation has a solution $\theta_{i_{0}}$ on $\left[\theta_{f}-2 \pi, \theta_{f}[\right.$ if and only if $\cos \left(\theta_{f}\right) \leq 0$. If this condition is satisfied, the solutions are:

$$
\begin{aligned}
& \theta_{f}^{-}=\theta_{f}-\arccos \left(-1-2 e \cos \left(\theta_{f}\right)\right), \\
& \hat{\theta}_{f}^{-}=\theta_{f}-2 \pi+\arccos \left(-1-2 e \cos \left(\theta_{f}\right)\right) .
\end{aligned}
$$

As previously explained, if $\theta_{f}-\theta_{0}<\pi$, then (56) has a unique solution on $\left[\theta_{f}-\pi, \theta_{f}\right]$, and has a solution on $\left[\theta_{0}, \theta_{f}\right]$ if and only if condition $1+2 e \cos \left(\theta_{f}\right)+\cos \left(\theta_{f}-\theta_{0}\right) \leq 0$ is satisfied. Otherwise, when $\theta_{f}-\theta_{0} \geq \pi$, then Equation (56) has a unique solution on $\left[\theta_{0}, \theta_{f}\right]$ if and only if:

$$
1+2 e \cos \left(\theta_{f}\right)+\cos \left(\theta_{f}-\theta_{0}\right)<0
$$

and two solutions if condition (58) does not hold.

Next, two cases are discussed: $\theta_{i_{0}} \in\left\{\theta_{0}^{+}, \theta_{f}^{-}\right\}$and $\theta_{i_{0}} \in\left\{\hat{\theta}_{0}^{+}, \hat{\theta}_{f}^{-}\right\}$, according to the notations defined by 54 and (57).
Case I: The results in the case where $\theta_{i_{0}} \in\left\{\theta_{0}^{+}, \theta_{f}^{-}\right\}$, are summarized in Proposition 3. To make the results clearer to the reader, it is important to emphasize that + is associated with $\#=0$ (initial impulse) while - is associated with $\#=f$ (final impulse) as is indicated by the notations (54) and (57).

Proposition 3: An optimal solution for the linearized impulsive out-of-plane rendezvous problem is a 2-impulse trajectory defined by the optimal locations $\left(\theta_{\sharp}, \theta_{\sharp}^{ \pm}\right)$and the associated optimal thrusts,

$$
\begin{aligned}
& \Delta V\left(\theta_{\sharp}\right)=\left(1+e \cos \left(\theta_{\sharp}\right)\right) \frac{\cos \left(\theta_{\sharp}^{ \pm}\right) z_{f_{1}}+\sin \left(\theta_{\sharp}^{ \pm}\right) z_{f_{2}}}{\sin \left(\theta_{\sharp}^{ \pm}-\theta_{\sharp}\right)}, \\
& \Delta V\left(\theta_{\sharp}^{ \pm}\right)=-\left(1+e \cos \left(\theta_{\sharp}^{ \pm}\right)\right) \frac{\cos \left(\theta_{\sharp}\right) z_{f_{1}}+\sin \left(\theta_{\sharp}\right) z_{f_{2}}}{\sin \left(\theta_{\sharp}^{ \pm}-\theta_{\sharp}\right)} .
\end{aligned}
$$

With $\sharp=0$ if the conditions:

$$
\begin{gathered}
d_{\theta}<\pi \text { and } \sin \left(\theta_{0}\right)<\sqrt{1-e^{2}} \\
\text { and } 1+2 e \cos \left(\theta_{0}\right)+\cos \left(\theta_{f}-\theta_{0}\right) \leq 0
\end{gathered}
$$

$$
\text { and } \varepsilon_{0}=\varepsilon_{f} \text { or }\left\{\begin{array}{l}
\varepsilon_{0}=-\varepsilon_{f} \\
\left|\vec{z}_{f}\right|+\left(2 e\left|\vec{z}_{f}\right|-\varepsilon_{0} z_{f_{2}}\right) \cos \left(\theta_{0}\right) \\
+\varepsilon_{0} z_{f_{1}} \sin \left(\theta_{0}\right) \leq 0
\end{array}\right.
$$

or

$$
\begin{gathered}
\pi \leq d_{\theta}<2 \pi \\
\text { and } \sin \left(\theta_{0}\right)<\sqrt{1-e^{2}} \text { and } \cos \left(\theta_{0}\right) \leq 0 \\
\text { and }\left\{\begin{array}{c}
1-\cos \left(\theta_{f}-\theta_{0}\right)+2 \sin \left(\theta_{f}-\theta_{0}\right) g^{-}\left(\theta_{0}\right) \geq 0 \\
\left|\vec{z}_{f}\right|+\left(2 e\left|\vec{z}_{f}\right|-\varepsilon_{0} z_{f_{2}}\right) \cos \left(\theta_{0}\right)+\varepsilon_{0} z_{f_{1}} \sin \left(\theta_{0}\right) \leq 0 \\
\sin \left(\theta_{f}-\theta_{0}\right)+e\left(\sin \left(\theta_{f}\right)-\sin \left(\theta_{0}\right)\right) \\
+2\left(\cos \left(\theta_{f}-\theta_{0}\right)+e \cos \left(\theta_{0}\right)\right) g^{-}\left(\theta_{0}\right) \leq 0
\end{array}\right.
\end{gathered}
$$

are verified.

With $\sharp=f$ if the conditions

$$
\begin{gathered}
d_{\theta}<\pi \text { and } \sin \left(\theta_{f}\right)>-\sqrt{1-e^{2}} \\
\text { and } 1+2 e \cos \left(\theta_{f}\right)+\cos \left(\theta_{f}-\theta_{0}\right) \leq 0 \\
\text { and } \varepsilon_{0}=\varepsilon_{f} \text { or }\left\{\begin{array}{l}
\varepsilon_{0}=-\varepsilon_{f} \\
\left|\vec{z}_{f}\right|+\left(2 e\left|\vec{z}_{f}\right|-\varepsilon_{0} z_{f_{2}}\right) \cos \left(\theta_{f}\right) \\
+\varepsilon_{0} z_{f_{1}} \sin \left(\theta_{f}\right) \leq 0
\end{array}\right.
\end{gathered}
$$

or

$$
\pi \leq d_{\theta}<2 \pi
$$

and $\sin \left(\theta_{f}\right)>-\sqrt{1-e^{2}}$ and $\cos \left(\theta_{f}\right) \leq 0$ and $1-\cos \left(\theta_{f}-\theta_{0}\right)+2 \sin \left(\theta_{f}-\theta_{0}\right) g^{+}\left(\theta_{f}\right) \geq 0$

$$
\text { and }\left\{\begin{array}{l}
\left|\vec{z}_{f}\right|+\left(2 e\left|\vec{z}_{f}\right|+\varepsilon_{f} z_{f_{2}}\right) \cos \left(\theta_{f}\right)-\varepsilon_{f} z_{f_{1}} \sin \left(\theta_{f}\right) \leq 0 \\
\sin \left(\theta_{f}-\theta_{0}\right)+e\left(\sin \left(\theta_{f}\right)-\sin \left(\theta_{0}\right)\right) \\
\quad+2\left(\cos \left(\theta_{f}-\theta_{0}\right)+e \cos \left(\theta_{f}\right)\right) g^{+}\left(\theta_{f}\right) \geq 0
\end{array}\right.
$$

are verified.

The optimal Lagrange multipliers are given by:

$$
\begin{aligned}
\lambda_{1}= & \varepsilon_{\sharp}\left( \pm \sin \left(\theta_{\sharp}\right)\left(1+2 e \cos \left(\theta_{\sharp}\right)\right.\right. \\
& \left.-2 \cos \left(\theta_{\sharp}\right) \sqrt{-e \cos \left(\theta_{\sharp}\right)\left(1+e \cos \left(\theta_{\sharp}\right)\right)}\right), \\
\lambda_{2}= & \varepsilon_{\sharp}\left( \pm e \mp \cos \left(\theta_{\sharp}\right)\left(1+e \cos \left(\theta_{\sharp}\right)\right.\right. \\
& \left.-2 \sin \left(\theta_{\sharp}\right) \sqrt{-e \cos \left(\theta_{\sharp}\right)\left(1+2 e \cos \left(\theta_{\sharp}\right)\right)}\right),
\end{aligned}
$$


while the optimal primer vector is defined by:

$$
p(\theta)=p\left(\theta_{\sharp}^{ \pm}\right) \frac{\cos \left(\theta-\theta_{\sharp}^{ \pm}\right)+e \cos (\theta)}{1+e \cos (\theta)} .
$$

Case II: The results in the case where $\theta_{i_{0}} \in\left\{\hat{\theta}_{0}^{+}, \hat{\theta}_{f}^{-}\right\}$, are summarized in Proposition 4. To make the results clearer to the reader, it is important to emphasize that + is associated with $\#=0$ (initial impulse) while - is associated with $\#=f$ (final impulse) as indicated by the notations (54) and (57).

Proposition 4: An optimal solution for the linearized impulsive out-of-plane rendezvous problem is a 2-impulse trajectory defined by the optimal locations $\left(\theta_{\sharp}, \hat{\theta}_{\sharp}^{ \pm}\right)$and the associated optimal thrusts given by (59).

With $\sharp=0$ if the conditions

$$
\begin{aligned}
& \pi \leq d_{\theta}<2 \pi \\
& \text { and } \sin \left(\theta_{0}\right)<-\sqrt{1-e^{2}} \text { and } \cos \left(\theta_{0}\right) \leq 0
\end{aligned}
$$

and $\left\{\begin{aligned} 1-\cos \left(\theta_{f}-\theta_{0}\right)-2 \sin \left(\theta_{f}-\theta_{0}\right) g^{+}\left(\theta_{0}\right) & \geq 0 \\ 1+2 e \cos \left(\theta_{0}\right)+\cos \left(\theta_{f}-\theta_{0}\right) & \geq 0 \\ \left|\vec{z}_{f}\right|+\left(2 e\left|\vec{z}_{f}\right|+\varepsilon_{0} z_{f_{2}}\right) \cos \left(\theta_{0}\right)-\varepsilon_{0} z_{f_{1}} \sin \left(\theta_{0}\right) & \leq 0 \\ \sin \left(\theta_{f}-\theta_{0}\right)+e\left(\sin \left(\theta_{f}\right)-\sin \left(\theta_{0}\right)\right) & \\ -2\left(\cos \left(\theta_{f}-\theta_{0}\right)+e \cos \left(\theta_{0}\right)\right) g^{+}\left(\theta_{0}\right) & \leq 0\end{aligned}\right.$

are verified.

With $\sharp=f$ if the conditions

$$
\pi \leq d_{\theta}<2 \pi
$$$$
\text { and } \sin \left(\theta_{f}\right)>\sqrt{1-e^{2}} \text { and } \cos \left(\theta_{f}\right) \leq 0
$$

and $\left\{\begin{aligned} & 1-\cos \left(\theta_{f}-\theta_{0}\right)-2 \sin \left(\theta_{f}-\theta_{0}\right) g^{-}\left(\theta_{f}\right) \geq 0 \\ & 1+2 e \cos \left(\theta_{f}\right)+\cos \left(\theta_{f}-\theta_{0}\right) \geq 0 \\ &\left|\vec{z}_{f}\right|+\left(2 e\left|\vec{z}_{f}\right|-\varepsilon_{f} z_{f_{2}}\right) \cos \left(\theta_{f}\right)+\varepsilon_{f} z_{f_{1}} \sin \left(\theta_{f}\right) \leq 0 \\ & \sin \left(\theta_{f}-\theta_{0}\right)+e\left(\sin \left(\theta_{f}\right)-\sin \left(\theta_{0}\right)\right) \\ &-2\left(\cos \left(\theta_{f}-\theta_{0}\right)+e \cos \left(\theta_{f}\right)\right) g^{-}\left(\theta_{f}\right) \leq 0\end{aligned}\right.$

are verified. The optimal Lagrange multipliers are given by:

$$
\begin{aligned}
\lambda_{1}= & \varepsilon_{\sharp}\left(\mp \sin \left(\theta_{\sharp}\right)\left(1+2 e \cos \left(\theta_{\sharp}\right)\right)\right. \\
& \left.-2 \cos \left(\theta_{\sharp}\right) \sqrt{-e \cos \left(\theta_{\sharp}\right)\left(1+e \cos \left(\theta_{\sharp}\right)\right)}\right) \\
\lambda_{2}= & \varepsilon_{\sharp}\left(\mp e \pm \cos \left(\theta_{\sharp}\right)\left(1+e \cos \left(\theta_{\sharp}\right)\right)\right. \\
& \left.-2 \sin \left(\theta_{\sharp}\right) \sqrt{-e \cos \left(\theta_{\sharp}\right)\left(1+2 e \cos \left(\theta_{\sharp}\right)\right)}\right)
\end{aligned}
$$

while the optimal primer vector is defined by:

$$
p(\theta)=p\left(\hat{\theta}_{\sharp}^{ \pm}\right) \frac{\cos \left(\theta-\hat{\theta}_{\sharp}^{ \pm}\right)+e \cos (\theta)}{1+e \cos (\theta)} .
$$

\section{Boundary solutions}

In this section, the case of optimal solutions with no interior impulse is discussed. In that case, the norm of the primer vector can reach 1 only at $\theta_{0}$ and $\theta_{f}$, which can occur only if $\theta_{f}-\theta_{0}<2 \pi$. The objective equation given by the optimality condition (14) leads to make the difference between two cases: $\theta_{f}-\theta_{0} \neq \pi$ and $\theta_{f}-\theta_{0}=\pi$.
Case I: $\theta_{f}-\theta_{0} \neq \pi$ :

Proposition 5: An optimal solution for the linearized impulsive out-of-plane rendezvous problem is a 2-impulse trajectory defined by the optimal locations $\left(\theta_{0}, \theta_{f}\right)$ and the associated optimal thrusts,

$$
\begin{aligned}
& \Delta V\left(\theta_{0}\right)=\left(1+e \cos \left(\theta_{0}\right)\right) \frac{\cos \left(\theta_{f}\right) z_{f_{1}}+\sin \left(\theta_{f}\right) z_{f_{2}}}{\sin \left(\theta_{f}-\theta_{0}\right)}, \\
& \Delta V\left(\theta_{f}\right)=-\left(1+e \cos \left(\theta_{f}\right)\right) \frac{\cos \left(\theta_{0}\right) z_{f_{1}}+\sin \left(\theta_{0}\right) z_{f_{2}}}{\sin \left(\theta_{f}-\theta_{0}\right)},
\end{aligned}
$$

if the conditions

$d_{\theta}<\pi$ and $\left(\left(\sin \left(\theta_{0}\right)<\sqrt{1-e^{2}}\right)\right.$ or $\left.\left(\sin \left(\theta_{f}\right)>-\sqrt{1-e^{2}}\right)\right)$

and

$$
\varepsilon_{0}=\varepsilon_{f} \text { and }\left\{\begin{array}{l}
1+2 e \cos \left(\theta_{f}\right)+\cos \left(\theta_{f}-\theta_{0}\right) \geq 0 \\
1+2 e \cos \left(\theta_{0}\right)+\cos \left(\theta_{f}-\theta_{0}\right) \geq 0 \\
\cos \left(\theta_{f}\right) z_{f_{1}}+\sin \left(\theta_{f}\right) z_{f_{2}} \neq 0 \\
\cos \left(\theta_{0}\right) z_{f_{1}}+\sin \left(\theta_{0}\right) z_{f_{2}} \neq 0
\end{array}\right.
$$

are verified and for which the optimal Lagrange multipliers are given by:

$$
\begin{aligned}
& \lambda_{1}=-\varepsilon_{0} \frac{\cos \left(\theta_{f}\right)+\cos \left(\theta_{0}\right)+2 e \cos \left(\theta_{0}\right) \cos \left(\theta_{f}\right)}{\sin \left(\theta_{f}-\theta_{0}\right)}, \\
& \lambda_{2}=-\varepsilon_{0} \frac{\sin \left(\theta_{f}\right)+\sin \left(\theta_{0}\right)+e \sin \left(\theta_{f}+\theta_{0}\right)}{\sin \left(\theta_{f}-\theta_{0}\right)},
\end{aligned}
$$

while the optimal primer vector is defined by:

$$
\begin{aligned}
p(\theta)= & \varepsilon_{0} \frac{\left(1+e \cos \left(\theta_{f}\right)\right) \sin \left(\theta-\theta_{0}\right)}{\sin \left(\theta_{f}-\theta_{0}\right)(1+e \cos (\theta))} \\
& -\varepsilon_{0} \frac{\sin \left(\theta_{f}-\theta\right)\left(1+e \cos \left(\theta_{0}\right)\right)}{\sin \left(\theta_{f}-\theta_{0}\right)(1+e \cos (\theta))},
\end{aligned}
$$

or

$$
\begin{gathered}
\pi<d_{\theta}<2 \pi \text { and } \varepsilon_{0}=-\varepsilon_{f} \\
\text { and }\left\{\begin{array}{l}
\cos \left(\theta_{f}\right) z_{f_{1}}+\sin \left(\theta_{f}\right) z_{f_{2}} \neq 0 \\
\cos \left(\theta_{0}\right) z_{f_{1}}+\sin \left(\theta_{0}\right) z_{f_{2}} \neq 0 \\
\frac{\left|\lambda_{2}\right|\left(1+Q^{2}\right)}{\sqrt{1+Q^{2}\left(1-e^{2}\right)+e}}<1
\end{array}\right.
\end{gathered}
$$

are verified and for which the optimal Lagrange multipliers are given by:

$\lambda_{1}=\varepsilon_{f} \frac{\cos \left(\theta_{f}\right)-\cos \left(\theta_{0}\right)}{\sin \left(\theta_{f}-\theta_{0}\right)}, \lambda_{2}=\varepsilon_{f}\left(\frac{\sin \left(\theta_{f}\right)-\sin \left(\theta_{0}\right)}{\sin \left(\theta_{f}-\theta_{0}\right)}+e\right)$,

while the optimal primer vector is defined by:

$$
p(\theta)=-\varepsilon_{f} \frac{\sin \left(\theta-\theta_{0}\right)+\sin \left(\theta_{f}-\theta\right)+e \cos \left(\theta_{0}\right)}{\sin \left(\theta_{f}-\theta_{0}\right)(1+e \cos (\theta))},
$$

or

$$
\pi<d_{\theta}<2 \pi \text { and } \varepsilon_{0}=\varepsilon_{f}
$$




$$
\begin{aligned}
& \text { and } \\
& \left\{\begin{array}{l}
1+2 e \cos \left(\theta_{f}\right)+\cos \left(\theta_{f}-\theta_{0}\right) \leq 0 \\
1+2 e \cos \left(\theta_{0}\right)+\cos \left(\theta_{f}-\theta_{0}\right) \leq 0 \\
\cos \left(\theta_{f}\right) z_{f_{1}}+\sin \left(\theta_{f}\right) z_{f_{2}} \neq 0 \\
\cos \left(\theta_{0}\right) z_{f_{1}}+\sin \left(\theta_{0}\right) z_{f_{2}} \neq 0 \\
\text { and } \\
\left\{\begin{array}{l}
\left|\sin \left(\theta_{0}\right)\right|<\sqrt{1-e^{2}} \\
\left(e+\cos \left(\theta_{0}\right)\right)\left(e+\cos \left(\theta_{f}\right)\right)<0
\end{array}\right. \\
\text { or } \begin{array}{l}
\sin \left(\theta_{0}\right)<-\sqrt{1-e^{2}} \\
\sin \left(\theta_{f}\right)>-\sqrt{1-e^{2}}
\end{array}
\end{array}\right.
\end{aligned}
$$

are verified and for which the optimal Lagrange multipliers are given by:

$$
\begin{aligned}
& \lambda_{1}=\varepsilon_{0} \frac{\cos \left(\theta_{f}\right)+\cos \left(\theta_{0}\right)+2 e \cos \left(\theta_{0}\right) \cos \left(\theta_{f}\right)}{\sin \left(\theta_{f}-\theta_{0}\right)}, \\
& \lambda_{2}=\varepsilon_{0} \frac{\sin \left(\theta_{f}\right)+\sin \left(\theta_{0}\right)+e \sin \left(\theta_{f}+\theta_{0}\right)}{\sin \left(\theta_{f}-\theta_{0}\right)},
\end{aligned}
$$

while the optimal primer vector is defined by:

$$
\begin{aligned}
p(\theta)= & -\varepsilon_{0} \frac{\left(1+e \cos \left(\theta_{f}\right)\right) \sin \left(\theta-\theta_{0}\right)}{\sin \left(\theta_{f}-\theta_{0}\right)(1+e \cos (\theta))} \\
& +\varepsilon_{0} \frac{\sin \left(\theta_{f}-\theta\right)\left(1+e \cos \left(\theta_{0}\right)\right.}{\sin \left(\theta_{f}-\theta_{0}\right)(1+e \cos (\theta))} .
\end{aligned}
$$

Case II: $\theta_{f}-\theta_{0}=\pi$ :

Corollary 2: When $d_{\theta}=\pi$ and $\theta_{0}=-\frac{\pi}{2}+k \pi, k \in \mathbb{Z}$, the optimal solution may be concentrated on one boundary impulse or scattered in two boundary impulses. In that case, there may exist an infinite number of optimal Lagrange multipliers $\left(-\varepsilon_{1}, \lambda_{2}\right),\left|\lambda_{2}\right| \leq e$, and the optimal directions and amplitudes of thrust are characterized by:

$$
\frac{\Delta V\left(\theta_{0}\right)}{\left|\Delta V\left(\theta_{0}\right)\right|}=\varepsilon_{1} \quad \text { and } \quad \frac{\Delta V\left(\theta_{f}\right)}{\left|\Delta V\left(\theta_{f}\right)\right|}=-\varepsilon_{1},
$$

The optimal consumption is given by: $\left|\Delta V\left(\theta_{0}\right)\right|+\left|\Delta V\left(\theta_{f}\right)\right|=$ $\left|\vec{z}_{f}\right|$.

\section{E. Summary of solutions and conditions}

The solutions derived in the previous subsections are quite complex due to the different cases and conditions that have to be considered but a systematic simple procedure may be defined to compute the optimal solution. Two tables are now given to help the reader to grasp the essentials of the paper and to design the associated algorithm providing one optimal solution in every case. Table I] summarizes the conditions to be met for each type of solution depending on the duration of the rendezvous. The first parameter to be checked is the duration $d_{\theta}$ of the rendezvous. For a given duration $d_{\theta}$, a column of the Table I is identified and one has to check the exclusive conditions involving the parameters $\theta_{0}, \theta_{f}, e$ and $\vec{z}_{f}$ in the chosen column. These tests involve only simple algebraic operations (additions and multiplications), evaluation of trigonometric and inverse trigonometric functions and sign tests. Once the case to be dealt with is known, Table $\Pi$ gives the corresponding fuel-optimal closed-form solutions in terms of their velocity increments and the location of the optimal impulses. Once again, only simple algebraic operations and evaluation of trigonometric and inverse trigonometric functions are involved.

In the Table I. n.a. is the abbreviation of not applicable.

\section{NUMERICAL EXAMPLES}

In this section, four different examples are presented for comparison's sake with two existing results borrowed from the state of the art: first, the standard 2-impulse solution, where the two thrusts are located at the beginning and at the end of the rendezvous. In addition, the optimal solution is compared to the recent method proposed in [4]. The main ingredients of the two methods are recalled below.

- Standard 2-impulse solution: The locations of the two impulses are defined as $\theta_{0}$ and $\theta_{f}$ and therefore, the computation of the DVs amounts to solve the linear system defined by (14):

$$
\frac{R\left(\theta_{0}\right)}{r\left(\theta_{0}\right)} \Delta V_{1}+\frac{R\left(\theta_{f}\right)}{r\left(\theta_{f}\right)} \Delta V_{2}=\vec{z}_{f},
$$

and whose solution is given by:

$$
\begin{aligned}
& \Delta V_{1}=\left(1+e \cos \left(\theta_{0}\right)\right) \frac{\cos \left(\theta_{f}\right) z_{f_{1}}+\sin \left(\theta_{f}\right) z_{f_{2}}}{\sin \left(\theta_{f}-\theta_{0}\right)}, \\
& \Delta V_{2}=-\left(1+e \cos \left(\theta_{f}\right)\right) \frac{\cos \left(\theta_{0}\right) z_{f_{1}}+\sin \left(\theta_{0}\right) z_{f_{2}}}{\sin \left(\theta_{f}-\theta_{0}\right)} .
\end{aligned}
$$

Note that this solution appears to be optimal when the conditions on $d_{\theta}, e, \vec{z}_{f}, \theta_{0}$ and $\theta_{f}$ of case D in Table [I] are met.

- 1-impulse solution: In [4], a 1-impulse strategy is defined based on the use of a particular set of relative orbital parameters. Applying the proposed strategy with the cartesian relative positions and velocities, the possible locations of the only impulse are given by:

$$
\theta_{k}=\arctan \left(-\frac{z_{f_{1}}}{z_{f_{2}}}\right)+k \pi, k=0,1, \cdots .
$$

Among all $\theta_{k}$ s computed with 82 , choose the one leading to the minimum-fuel impulse, calculated by solving the linear system (14):

$$
\frac{R\left(\theta_{k}\right)}{r\left(\theta_{k}\right)} \Delta V\left(\theta_{k}\right)=\vec{z}_{f}
$$

and whose solution is given by:

$$
\Delta V\left(\theta_{k}\right)=-z_{f_{1}} \frac{1+e \cos \left(\theta_{k}\right)}{\sin \left(\theta_{k}\right)}=z_{f_{2}} \frac{1+e \cos \left(\theta_{k}\right)}{\cos \left(\theta_{k}\right)}
$$

If the procedure gives the minimum-fuel 1-impulse solution, there is no guarantee that this solution is optimal when varying the possible number of impulses except when the conditions described in Table I for the cases $\mathrm{B}_{1}$ and $\mathrm{B}_{2}$ are fulfilled. 


\begin{tabular}{|c|c|c|c|}
\hline Case $\quad$ duration & $d_{\theta}<\pi$ & $\pi<d_{\theta}<2 \pi$ & $d_{\theta} \geq 2 \pi$ \\
\hline $\begin{array}{c}\mathrm{A} \\
2 \text { int. imp. }\end{array}$ & $\begin{array}{c}e\left|\vec{z}_{f}\right|>\left|z_{f_{2}}\right| \text { and } \sin \left(\theta_{0}\right) \geq \sqrt{1-e^{2}} \\
\text { and } \sin \left(\theta_{f}\right) \leq-\sqrt{1-e^{2}}\end{array}$ & $e\left|\vec{z}_{f}\right|>\left|z_{f_{2}}\right|$ and 34 & $e\left|\vec{z}_{f}\right|>\left|z_{f_{2}}\right|$ \\
\hline $\begin{array}{l}B_{1} \\
1 \text { int. imp. }\end{array}$ & $\begin{array}{c}e\left|\vec{z}_{f}\right| \leq \varepsilon_{0} z_{f_{2}} \text { and } \varepsilon_{f}=-\varepsilon_{0} \\
\text { or } \\
e\left|\vec{z}_{f}\right|>\varepsilon_{0} z_{f_{2}} \text { and } \varepsilon_{f}=-\varepsilon_{0} \text { and (43) }\end{array}$ & $\begin{array}{c}e\left|\vec{z}_{f}\right| \leq \varepsilon_{0} z_{f_{2}} \text { and } \varepsilon_{f}=-\varepsilon_{0} \\
\text { or } \\
e\left|\vec{z}_{f}\right|>\varepsilon_{0} z_{f_{2}} \text { and } \varepsilon_{f}=-\varepsilon_{0} \text { and (43) }\end{array}$ & n.a. \\
\hline $\begin{array}{l}B_{2} \\
1 \text { int. imp. }\end{array}$ & n.a. & $\begin{array}{c}e\left|\vec{z}_{f}\right| \leq \varepsilon_{0} z_{f_{2}} \text { and } \varepsilon_{f}=\varepsilon_{0} \\
\text { or } \\
e\left|\vec{z}_{f}\right|>\varepsilon_{0} z_{f_{2}} \text { and } \varepsilon_{f}=\varepsilon_{0} \text { and } 46\end{array}$ & $e\left|\vec{z}_{f}\right|<\left|z_{f_{2}}\right|$ \\
\hline $\begin{array}{c}C_{1} \\
1 \text { init. } \\
1 \text { int. imp. }\end{array}$ & $\begin{array}{c}\sin \left(\theta_{0}\right)<\sqrt{1-e^{2}} \text { and }(60) \text { and } \\
1+2 e \cos \left(\theta_{0}\right)+\cos \left(\theta_{f}-\theta_{0}\right) \leq 0\end{array}$ & $\begin{array}{c}\sin \left(\theta_{0}\right)<\sqrt{1-e^{2}} \\
\text { and } \\
\cos \left(\theta_{0}\right) \leq 0 \text { and } 61\end{array}$ & n.a. \\
\hline $\begin{array}{l}C_{2} \\
1 \text { int. imp. } \\
1 \text { final imp. }\end{array}$ & $\begin{array}{l}\left.\sin \left(\theta_{f}\right)>-\sqrt{1-e^{2}} \text { and } 62\right) \text { and } \\
1+2 e \cos \left(\theta_{f}\right)+\cos \left(\theta_{f}-\theta_{0}\right) \leq 0\end{array}$ & $\begin{aligned} & \sin \left(\theta_{f}\right)>-\sqrt{1-e^{2}} \\
& \text { and } \\
& \cos \left(\theta_{f}\right) \leq 0 \text { and } 63\end{aligned}$ & n.a. \\
\hline $\begin{array}{l}\text { C } C_{3} \\
1 \text { init. imp. } \\
1 \text { int. imp. }\end{array}$ & n.a. & $\begin{aligned} \sin \left(\theta_{0}\right) & <-\sqrt{1-e^{2}} \\
& \text { and } \\
\cos \left(\theta_{0}\right) \leq 0 \text { and } & \end{aligned}$ & n.a. \\
\hline $\begin{array}{c}C_{4} \\
1 \text { int. imp. } \\
1 \text { final imp. }\end{array}$ & n.a. & $\begin{aligned} & \sin \left(\theta_{f}\right)>\sqrt{1-e^{2}} \\
& \text { and } \\
& \cos \left(\theta_{f}\right) \leq 0 \text { and }(67)\end{aligned}$ & n.a. \\
\hline $\begin{array}{l}D \\
1 \text { init. imp. } \\
1 \text { final imp. }\end{array}$ & $\begin{array}{c}\sin \left(\theta_{0}\right)<\sqrt{1-e^{2}} \text { and } 71 \\
\text { or } \\
\sin \left(\theta_{f}\right)>-\sqrt{1-e^{2}} \text { and } 71\end{array}$ & $\begin{array}{c}\varepsilon_{0}=-\varepsilon_{f} \text { and } \overline{747} \\
\quad \text { or } \\
\varepsilon_{0}=\varepsilon_{f} \text { and } 77\end{array}$ & n.a. \\
\hline
\end{tabular}

TABLE I

SUMMARY OF THE CONDITIONS/SOLUTIONS DEPENDING ON THE DURATION OF THE RENDEZVOUS

\begin{tabular}{|c|c|c|}
\hline Solutions & Velocity Increments & Impulse Locations \\
\hline $\begin{array}{l}\text { A } \\
2 \text { int. imp. }\end{array}$ & $\begin{array}{l}\Delta V\left(\theta_{+}\right)=\frac{\sqrt{1-e^{2}}}{2 e}\left(-e z_{f_{1}}-\sqrt{1-e^{2}} z_{f_{2}}\right) \\
\Delta V\left(\theta_{-}\right)=\frac{\sqrt{1-e^{2}}}{2 e}\left(+e z_{f_{1}}-\sqrt{1-e^{2}} z_{f_{2}}\right)\end{array}$ & 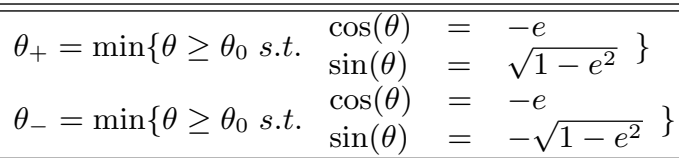 \\
\hline $\begin{array}{c}B_{1} \\
1 \text { int. imp. }\end{array}$ & $\Delta V\left(\theta_{i 0}\right)=-\varepsilon_{0}\left|\vec{z}_{f}\right|+e z_{f_{2}}$ & $\cos \left(\theta_{i_{0}}\right)=-\varepsilon_{0} \frac{z_{f_{2}}}{\left|\vec{z}_{f}\right|}, \quad \sin \left(\theta_{i_{0}}\right)=\varepsilon_{0} \frac{z_{f_{1}}}{\left|\vec{z}_{f}\right|}$ \\
\hline $\begin{array}{c}B_{2} \\
1 \text { int. imp. }\end{array}$ & $\Delta V\left(\theta_{i 2}\right)=-\varepsilon_{2}\left|\vec{z}_{f}\right|+e z_{f_{2}}$ & $\cos \left(\theta_{i_{2}}\right)=-\varepsilon_{2} \frac{z_{f_{2}}}{\left|\vec{z}_{f}\right|}, \quad \sin \left(\theta_{i_{2}}\right)=\varepsilon_{2} \frac{z_{f_{1}}}{\left|\vec{z}_{f}\right|}$ \\
\hline $\begin{array}{l}\quad C_{1} \\
1 \text { init. imp. } \\
1 \text { int. imp. }\end{array}$ & $\begin{array}{l}\Delta V\left(\theta_{0}\right)=\left(1+e \cos \left(\theta_{0}\right)\right) \frac{\cos \left(\theta_{0}^{+}\right) z_{f_{1}}+\sin \left(\theta_{0}^{+}\right) z_{f_{2}}}{\sin \left(\theta_{0}^{+}-\theta_{0}\right)} \\
\Delta V\left(\theta_{0}^{+}\right)=-\left(1+e \cos \left(\theta_{0}^{+}\right)\right) \frac{\cos \left(\theta_{0}\right) z_{f_{1}}+\sin \left(\theta_{0}\right) z_{f_{2}}}{\sin \left(\theta_{0}^{+}-\theta_{0}\right)}\end{array}$ & $\begin{array}{l}\theta_{0} \text { and } \\
\theta_{0}^{+}=\theta_{0}+\arccos \left(-1-2 e \cos \left(\theta_{0}\right)\right)\end{array}$ \\
\hline $\begin{array}{l}\quad C_{2} \\
1 \text { int. Imp. } \\
1 \text { final imp. }\end{array}$ & $\begin{array}{l}\Delta V\left(\theta_{f}\right)=\left(1+e \cos \left(\theta_{f}\right)\right) \frac{\cos \left(\theta_{f}^{-}\right) z_{f_{1}}+\sin \left(\theta_{f}^{-}\right) z_{f_{2}}}{\sin \left(\theta_{f}^{-}-\theta_{f}\right)} \\
\Delta V\left(\theta_{f}^{-}\right)=-\left(1+e \cos \left(\theta_{f}^{-}\right)\right) \frac{\cos \left(\theta_{f}\right) z_{f_{1}}+\sin \left(\theta_{f}\right) z_{f_{2}}}{\sin \left(\theta_{f}^{-}-\theta_{f}\right)}\end{array}$ & $\begin{array}{l}\theta_{f}^{-}=\theta_{f}-\arccos \left(-1-2 e \cos \left(\theta_{f}\right)\right) \\
\text { and } \theta_{f}\end{array}$ \\
\hline $\begin{array}{l}\quad C_{3} \\
1 \text { init. imp. } \\
1 \text { int. imp. }\end{array}$ & $\begin{array}{l}\Delta V\left(\theta_{0}\right)=\left(1+e \cos \left(\theta_{0}\right)\right) \frac{\cos \left(\hat{\theta}_{0}^{+}\right) z_{f_{1}}+\sin \left(\hat{\theta}_{0}^{+}\right) z_{f_{2}}}{\sin \left(\hat{\theta}_{0}^{+}-\theta_{0}\right)} \\
\Delta V\left(\hat{\theta}_{0}^{+}\right)=-\left(1+e \cos \left(\hat{\theta}_{0}^{+}\right)\right) \frac{\cos \left(\theta_{0}\right) z_{f_{1}}+\sin \left(\theta_{0}\right) z_{f_{2}}}{\sin \left(\hat{\theta}_{0}^{+}-\theta_{\sharp}\right)}\end{array}$ & $\begin{array}{l}\theta_{0} \text { and } \\
\hat{\theta}_{0}^{+}=\theta_{0}+2 \pi-\arccos \left(-1-2 e \cos \left(\theta_{0}\right)\right)\end{array}$ \\
\hline $\begin{array}{l}\quad C_{4} \\
1 \text { int. imp. } \\
1 \text { final imp. }\end{array}$ & $\begin{array}{l}\Delta V\left(\theta_{f}\right)=\left(1+e \cos \left(\theta_{f}\right)\right) \frac{\cos \left(\hat{\theta}_{f}^{-}\right) z_{f_{1}}+\sin \left(\hat{\theta}_{f}^{-}\right) z_{f_{2}}}{\sin \left(\hat{\theta}_{f}^{-}-\theta_{f}\right)} \\
\Delta V\left(\hat{\theta}_{f}^{-}\right)=-\left(1+e \cos \left(\hat{\theta}_{f}^{-}\right)\right) \frac{\cos \left(\theta_{f}\right) z_{f_{1}}+\sin \left(\theta_{f}\right) z_{f_{2}}}{\sin \left(\hat{\theta}_{f}^{-}-\theta_{f}\right)}\end{array}$ & $\begin{array}{l}\hat{\theta}_{f}^{-}=\theta_{f}-2 \pi+\arccos \left(-1-2 e \cos \left(\theta_{f}\right)\right) \\
\text { and } \theta_{f}\end{array}$ \\
\hline $\begin{array}{l}\quad D \\
1 \text { init. imp. } \\
1 \text { final imp. }\end{array}$ & $\begin{array}{l}\Delta V\left(\theta_{0}\right)=\left(1+e \cos \left(\theta_{0}\right)\right) \frac{\cos \left(\theta_{f}\right) z_{f_{1}}+\sin \left(\theta_{f}\right) z_{f_{2}}}{\sin \left(\theta_{f}-\theta_{0}\right)} \\
\Delta V\left(\theta_{f}\right)=-\left(1+e \cos \left(\theta_{f}\right)\right) \frac{\cos \left(\theta_{0}\right) z_{f_{1}}+\sin \left(\theta_{0}\right) z_{f_{2}}}{\sin \left(\theta_{f}-\theta_{0}\right)}\end{array}$ & $\theta_{0}$ and $\theta_{f}$ \\
\hline
\end{tabular}

\section{TABLE II}

SUMMARY OF FUEL-OPTIMAL SOLUTIONS FOR EACH CASE 


\section{A. Example 1}

The first numerical example is based on the PROBA-3 mission whose main goals are to demonstrate the technologies required for Formation Flying of two spacecraft in highly elliptical orbit [19]. The necessary orbital elements and conditions for the out-of-plane rendezvous definition are given in Table III Two different initial state vectors $\vec{X}_{0_{1}}, \vec{X}_{0_{2}}$ and two different final time of rendezvous $\theta_{f_{1}}, \theta_{f_{2}}$ are considered while the other parameters defining the conditions of the rendezvous remain unchanged.

\begin{tabular}{|c|c|}
\hline$a[\mathrm{~m}]$ & 37039887 \\
\hline$e$ & 0.80621 \\
\hline$\theta_{0}[\mathrm{rad}]$ & 2.042 \\
\hline$\vec{X}_{0_{1}}^{T}[\mathrm{~m} \mathrm{~m} / \mathrm{s}]$ & {$\left[\begin{array}{cc}-5000 & 0.5\end{array}\right]$} \\
\hline$\vec{X}_{0_{2}}^{T}[\mathrm{~m} \mathrm{~m} / \mathrm{s}]$ & {$\left[\begin{array}{ll}-5000 & 0\end{array}\right]$} \\
\hline$\theta_{f_{1}}[\mathrm{rad}]$ & $3 \pi$ \\
\hline$\theta_{f_{2}}[\mathrm{rad}]$ & $4 \pi$ \\
\hline$\vec{X}_{f}^{T}[\mathrm{~m} \mathrm{~m} / \mathrm{s}]$ & {$\left[\begin{array}{ll}20 & 0.2\end{array}\right]$} \\
\hline \multicolumn{2}{|c|}{ TABLE III }
\end{tabular}

RENDEZVOUS PARAMETERS FOR PROBA-3 EXAMPLE: CASES 1 AND 2

Case 1: $\vec{X}_{0}=\vec{X}_{0_{1}}$ and $\theta_{f}=\theta_{f_{1}}$ : the duration of the rendezvous $d_{\theta}$ is greater than $2 \pi$ and therefore the conditions to be checked in order to identify which case will lead to the optimal solution, are given in the last column of Table II. Here, using the values of the parameters given in Table III, it

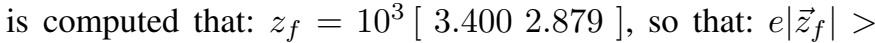
$\left|z_{f_{2}}\right|$, corresponding to the case A. The optimal solution is a 2-impulse solution with their location strictly in the interior of $\left[\theta_{0}, \theta_{f}\right]$. According to the first line of Table $[1]$ this solution is defined by its velocity increments and their optimal locations:

$$
\begin{aligned}
& \Delta V_{1}=\frac{\sqrt{1-e^{2}}}{2 e}\left(-e z_{f_{1}}-\sqrt{1-e^{2}} z_{f_{2}}\right)=0.6975[\mathrm{~m} / \mathrm{s}], \\
& \Delta V_{2}=\frac{\sqrt{1-e^{2}}}{2 e}\left(+e z_{f_{1}}-\sqrt{1-e^{2}} z_{f_{2}}\right)=0.1639[\mathrm{~m} / \mathrm{s}] \text {, } \\
& \theta_{1}=\min \left\{\theta \geq \theta_{0} \text { s.t. } \begin{array}{l}
\cos (\theta)=-e, \\
\sin (\theta)=\sqrt{1-e^{2}}
\end{array}\right\}=2.5085[\mathrm{rad}], \\
& \theta_{2}=\min \left\{\theta \geq \theta_{0} \text { s.t. } \begin{array}{l}
\cos (\theta)=-e, \\
\sin (\theta)=-\sqrt{1-e^{2}}
\end{array}\right\}=3.7747[\mathrm{rad}] .
\end{aligned}
$$

Applying respectively (84) for the proposed optimal solution (o.s.), 81) for the 2-impulse solution (2-i) and (82) and 83) for the 1-impulse strategy leads to the numerical optimal solution in Table IV. Figures 3(a), 3(b) and 4 respectively depict the three out-of-plane trajectories in the phase plane, the corresponding position and velocity trajectories and the primer vector attached to these particular solutions.
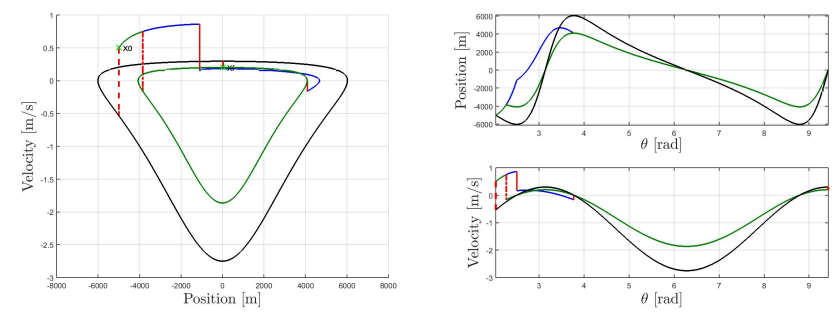

(a) Optimal (blue), 1-impulse (green) (b) Position and velocity vs true and standard 2-impulse (black) trajec- anomaly. tories in phase plane.

Fig. 3. Numerical Example: PROBA-3 (case 1). Note that the blue and green trajectories partially overlap.

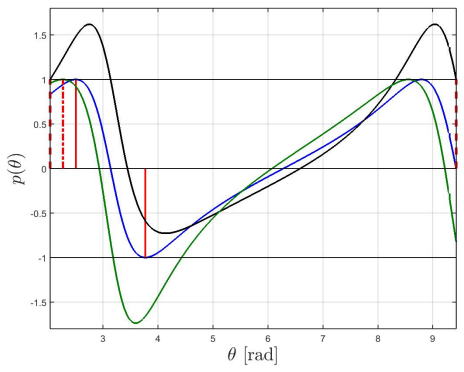

Fig. 4. Optimal (blue) and nonoptimal 2-impulse (black) and 1-impulse (green) primer vectors: PROBA-3 (case 1)

\begin{tabular}{|c|c|c|c|c|c|}
\hline & $\theta_{1}[\mathrm{rad}]$ & $\Delta V_{1}[\mathrm{~m} / \mathrm{s}]$ & $\theta_{2}[\mathrm{rad}]$ & $\Delta V_{2}[\mathrm{~m} / \mathrm{s}]$ & Cost $[\mathrm{m} / \mathrm{s}]$ \\
\hline o.s. & 2.5085 & -0.6975 & 3.7747 & 0.1639 & 0.8614 \\
\hline 2-i. & 2.042 & -1.0348 & $3 \pi$ & -0.095 & 1.1298 \\
\hline 1-i. & 2.27 & -0.9128 & - & - & 0.9128 \\
\hline \multicolumn{5}{|c}{ TABLE IV } \\
TAB
\end{tabular}

OPTIMAL SOLUTION WITH A MINIMUM NUMBER OF IMPULSES, STANDARD 2-IMPULSE SOLUTION AND 1-IMPULSE SOLUTION FOR CASE 1 OF PROBA-3 EXAMPLE.

Note that, since $d_{\theta}>2 \pi$, it is always possible to choose a different optimal solution with the same cost but scattered over the maximum number of impulsive maneuvers while preserving the optimal consumption as indicated by Remark 1. One 3-impulse optimal solution is given in Table V] Note also that $\theta_{3}=\theta_{1}+2 \pi$.

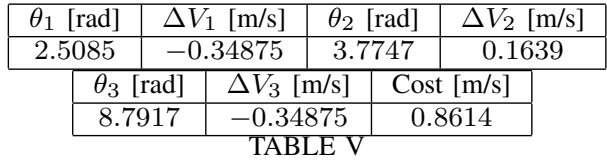

OPTIMAL SOLUTION SCATTERED OVER 3 IMPULSES FOR PROBA-3 EXAMPLE: CASE 1.

The choice between two equivalent minimum-fuel solution with a different number of thrusts (2-impulse solution and 3 -impulse solution) may be justified by different operational constraints. For instance, considering that a maximum velocity increment for this type of mission is set to $0.5 \mathrm{~m} / \mathrm{s}$, it is easily seen that the 2 -impulse optimal solution would not be eligible when the 3-impulse optimal solution would be. On the contrary, if some operational constraint has to be met in the second part of the rendezvous, preventing to fire again the engine, the 2-impulse mission could be preferable if it respects the maximum Delta- $\mathrm{V}$ allowed by the designer.

Case 2: $\vec{X}_{0}=\vec{X}_{0_{2}}$ and $\theta_{f}=\theta_{f_{2}}$ : When $\vec{X}_{0}=\vec{X}_{0_{2}}$ and $\theta_{f}=\theta_{f_{2}}$, the duration of the rendezvous is still such that $d_{\theta}>$ $2 \pi$. One has now: $z_{f}=10^{3}$ [ 1.7974 .714 ] and: $e\left|\vec{z}_{f}\right|<\left|z_{f_{2}}\right|$, corresponding to the case $B_{2}$ in Table I] Table II indicates that the optimal solution is an interior 1-impulse solution defined by:

$$
\begin{aligned}
& \Delta V_{1}=-\varepsilon_{2}\left|\vec{z}_{f}\right|+e z_{f_{2}}, \\
& \cos \left(\theta_{1}\right)=-\varepsilon_{2} \frac{z_{f_{2}}}{\left|\vec{z}_{f}\right|}, \sin \left(\theta_{1}\right)=\varepsilon_{2} \frac{z_{f_{1}}}{\left|\vec{z}_{f}\right|} .
\end{aligned}
$$

Here, applying the method from [4] leads to an identical solution and the comparison is made with the standard 2- 
impulse approach. The corresponding numerical solutions are given in Table $\mathrm{VI}$.

\begin{tabular}{|l|c|c|c|c|c|}
\hline & $\theta_{1}[\mathrm{rad}]$ & $\Delta V_{1}[\mathrm{~m} / \mathrm{s}]$ & $\theta_{2}[\mathrm{rad}]$ & $\Delta V_{2}[\mathrm{~m} / \mathrm{s}]$ & Cost $[\mathrm{m} / \mathrm{s}]$ \\
\hline o.s. & 2.777 & -0.5322 & - & - & 0.5322 \\
\hline 2-i. & 2.042 & -0.5470 & $4 \pi$ & 2.9341 & 3.481 \\
\hline \multicolumn{7}{|c|}{ TABLE VI }
\end{tabular}

OPTIMAL SOLUTION WITH A MINIMUM NUMBER OF IMPULSES, STANDARD 2-IMPULSE SOLUTION FOR CASE 2 OF PROBA-3 EXAMPLE.

The out-of-plane trajectory in the phase plane and relative position and velocity for each solution are given respectively in Figures 5(a) and 5(b) while the two corresponding primer vector histories is depicted in Figure 6
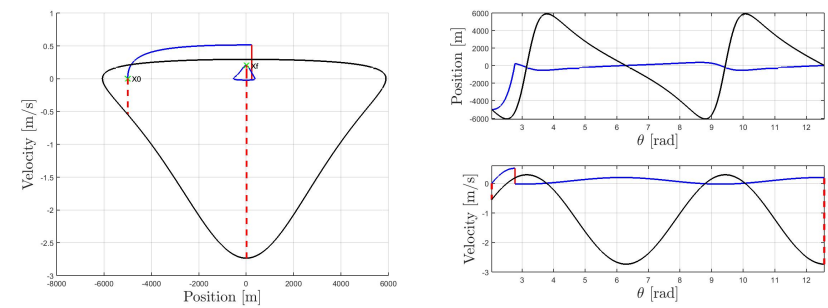

(a) Optimal (blue) and standard 2-(b) Position and velocity vs true impulse (black) trajectories in phase anomaly. plane.

Fig. 5. Numerical Example: PROBA-3 (case 2). Note that the blue and green trajectories partially overlap.

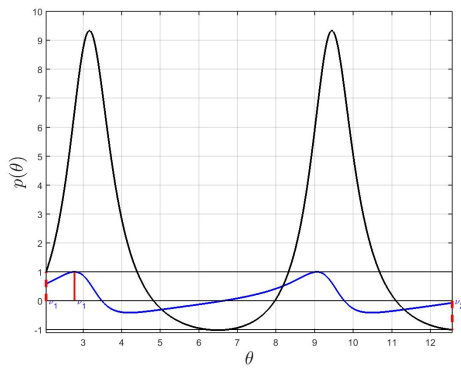

Fig. 6. Optimal (blue) and nonoptimal 2-impulses (black) primer vectors: PROBA-3 (case 2).

\section{B. Example 2}

Let us consider the numerical example borrowed from the reference [20], for which the target spacecraft is in the geostationary orbit transfer (GTO). It is a highly elliptical Earth orbit with apogee of $42.164 \mathrm{~km}$. The rendezvous characteristics are summarized in Table VII

\begin{tabular}{|c|c|}
\hline$a[\mathrm{~m}]$ & 24616000 \\
\hline$e$ & 0.73074 \\
\hline$\theta_{0}[\mathrm{rad}]$ & $0.1 \pi$ \\
\hline$\vec{X}_{0}^{T}[\mathrm{~m} \mathrm{~m} / \mathrm{s}]$ & {$\left[\begin{array}{cc}10000 & -3\end{array}\right]$} \\
\hline$\theta_{f_{1}}[\mathrm{rad}]$ & 5.2 \\
\hline$\theta_{f_{2}}[\mathrm{rad}]$ & {$\left[\begin{array}{ll}0 & 0\end{array}\right]$} \\
\hline$\vec{X}_{f}^{T}[\mathrm{~m} \mathrm{~m} / \mathrm{s}]$ & $10^{4}\left[\begin{array}{ll}-1.788 & 0.018\end{array}\right]$ \\
\hline$z_{f}$ & TABLE VII \\
\hline
\end{tabular}

RENDEZVOUS PARAMETERS: $[20$
Two different durations of rendezvous are considered. The first case is directly the one presented in [20] with $\pi<$ $d_{\theta_{1}}<2 \pi$ while the duration of the second rendezvous has been shortened, $d_{\theta_{2}}<\pi$, when the initial and final conditions remain unchanged.

Case 1: $\theta_{f}=5.2 \mathrm{rad} .:$ In this case, the optimal out-of-plane solution is a 2-impulse solution with initial and final coasting defined again as the case $\mathrm{A}$ in Table I. Here, the final coasting may be considered as a degenerate one since the chaser has reached the final conditions after the second maneuver.

\begin{tabular}{|c|c|c|c|c|c|}
\hline & $\theta_{1}[\mathrm{rad}]$ & $\Delta V_{1}[\mathrm{~m} / \mathrm{s}]$ & $\theta_{2}[\mathrm{rad}]$ & $\Delta V_{2}[\mathrm{~m} / \mathrm{s}]$ & Cost $[\mathrm{m} / \mathrm{s}]$ \\
\hline o.s. & 2.3902 & 3.1060 & 3.8930 & -3.1668 & 6.2728 \\
\hline 2-i. & $0.1 \pi$ & 7.5533 & 5.2 & -11.8696 & 19.4229 \\
\hline 1-i. & 1.5604 & -9.1196 & - & - & 9.1196 \\
\hline
\end{tabular}

OPTIMAL SOLUTION, STANDARD 2-IMPULSE SOLUTION AND 1-IMPULSE SOLUTION FOR CASE 1 OF GTO EXAMPLE.

The optimal solution may be compared to the standard 2impulse and 1-impulse solutions in Table VIII. It clearly shows the non-optimality of the last two plans of maneuvers. The standard 2-impulse solution is three times more expansive than the optimal one, when the 1-impulse plan uses up $45 \%$ more fuel than the optimal one. The phase plane, relative position and velocity trajectories may be compared in Figures 7(a) and 7(b) Finally, Figure 8 shows the three different primer vectors.
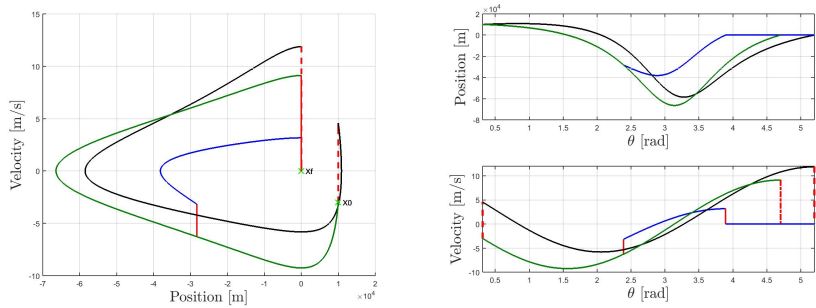

(a) Optimal (blue), 1-impulse (green) (b) Positions and velocities vs true and standard 2-impulse (black) trajec- anomaly. tories in phase plane.

Fig. 7. Numerical Example: GTO (case 1). Note that the blue and green trajectories partially overlap.

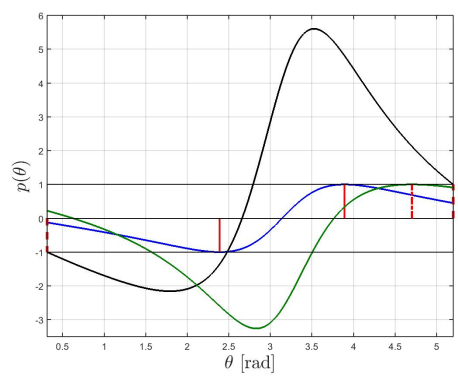

Fig. 8. Optimal (blue) and nonoptimal 2-impulses (black) primer vectors: GTO (case 1)

Case 2: $\theta_{f}=3$ rad.: The particular nature of the previous optimal solution is further illustrated by the following case where the duration of the rendezvous has been shortened resulting in a 2-impulse optimal solution with an initial coasting 
and a final impulse and detected as case $C_{2}$ in Table I] For this case, using formulae given in Table $\mathrm{II}$ in the corresponding row, the results given in Table $[\mathrm{IX}$ are obtained and may be compared to the two other approaches. The reduction of the duration of the rendezvous obviously results in increasing the consumption for all methods but it is important to notice that the consumption of the standard 2-impulse is particularly sensitive to the change of the duration of the rendezvous.

\begin{tabular}{|l|c|c|c|c|c|}
\hline & $\theta_{1}[\mathrm{rad}]$ & $\Delta V_{1}[\mathrm{~m} / \mathrm{s}]$ & $\theta_{2}[\mathrm{rad}]$ & $\Delta V_{2}[\mathrm{~m} / \mathrm{s}]$ & Cost $[\mathrm{m} / \mathrm{s}]$ \\
\hline o.s. & 1.8924 & 7.8311 & 3 & -0.9261 & 8.7572 \\
\hline 2 -i. & $0.1 \pi$ & 35.0842 & 3 & 5.4730 & 40.5572 \\
\hline 1 -i. & 1.5604 & 9.2591 & - & - & 9.2591 \\
\hline
\end{tabular}

OPTIMAL SOLUTION, STANDARD 2-IMPULSE SOLUTION AND 1-IMPULSE SOLUTION FOR CASE 2 OF GTO EXAMPLE.

The phase plane, relative position and velocity trajectories may be compared in Figures 9(a) and 9(b) Finally, Figure 10 shows the three different primer vectors.
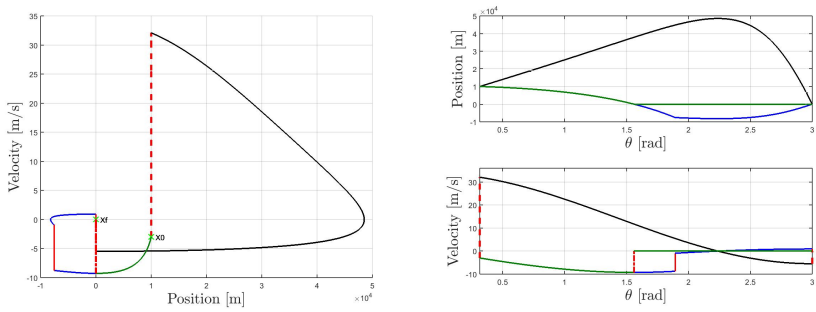

(a) Optimal (blue), 1-impulse (green) (b) Positions and velocities vs true and standard 2-impulse (black) trajec- anomaly. tories in phase plane.

Fig. 9. Numerical Example: GTO (case 2). Note that the blue and green trajectories partially overlap.

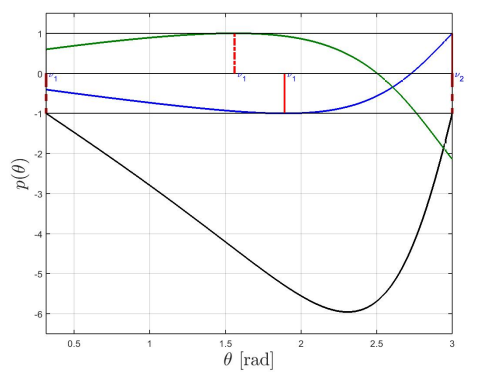

Fig. 10. Optimal (blue), nonoptimal 2-impulse (black) and 1-impulse (green) primer vectors: GTO (case 2)

\section{CONCLUSION}

The problem of time-fixed fuel-optimal out-of-plane elliptic rendezvous between spacecraft in a linear setting was still an open problem. This paper presents a new complete analytical closed-form solution to address this problem. It is mainly based on the so-called primer vector theory which is used to capture all possible optimal solutions for any duration and any boundary conditions of the rendezvous. The derivations of these results are based on the particular properties of the primer vector and on the thorough analysis of the possible primer vector candidates that have to meet the optimality conditions. Depending upon the duration of the rendezvous, conditions are derived to identify if the optimal solutions consist in one interior impulse, two interior impulses, one interior and one boundary impulses or one single boundary solution. In addition, it is shown that when the rendezvous may last more than one revolution, the designer has extra degrees of freedom allowing to split the optimal consumption over a maximum number of impulses that may be greater than the usual upper-bound of Neustadt. Despite its apparent complexity (high number of different cases and conditions), this analytical solution paves the way for onboard implementation in order to develop operational autonomy of future missions. Indeed, the optimal solutions are directly expressed in terms of the data of the problem (eccentricity, initial and final anomaly, boundary conditions) and do not require timeconsuming computations to be obtained.

In addition, it is worth noticing that similar analytical results are likely to be provided for non elliptic Keplerian reference orbits (parabolic $e=1$ and hyperbolic $e>1$ ) using identical derivations and might be an interesting topic in the future.

\section{APPENDIX}

The case where the rendezvous lasts more than $2 \pi$ is first considered. Consistent with the notation for the true anomalies $\theta_{+}$and $\theta_{-}$, let

$$
N_{ \pm}=\max \left\{i \in \mathbb{N}_{>0}: \theta_{ \pm}+2(i-1) \pi \leq \theta_{f}\right\} .
$$

Due to optimality conditions $(12)$ and $(13)$, the optimal impulses could only occur at the extrema locations in $\left[\theta_{0}, \theta_{f}\right]$ of the primer vector. From the extremum ratio (22), it is observed that $\theta_{+}$and $\theta_{-}$are necessarily the locations in $\left[\theta_{0}, \theta_{0}+2 \pi\right)$ of the extrema of the primer vector $\left|p\left(\theta_{ \pm}\right)\right|=1$ and $\frac{\mathrm{d} p}{\mathrm{~d} \theta}\left(\theta_{ \pm}\right)=0$. Any optimal solution with two interior impulses per period, is composed of at most $N_{+}+N_{-}$optimal impulses, located at $\theta_{i} \in\left\{\theta_{i}^{+}: i=1, \cdots, N_{+}\right\} \cup\left\{\theta_{i}^{-}: i=1, \cdots, N_{-}\right\}$where $\theta_{i}^{ \pm}=\theta_{ \pm}+2(i-1) \pi, i=1, \cdots, N_{ \pm}$. By the way, from 18 and the conditions on the primer vector and its derivative, the second optimal Lagrange multiplier is obtained as $\lambda_{2}^{*}=0$. Hence, $p\left(\theta_{+}\right)=-p\left(\theta_{-}\right)=-\frac{\lambda_{1}}{\sqrt{1-e^{2}}}$ due to the definition of $\theta_{+}$and $\theta_{-}$given by (32). The optimality condition (11) leads to the optimal directions of thrust: for all $i=1, \cdots, N_{+}$,

$$
\frac{\Delta V\left(\theta_{i}^{+}\right)}{\left|\Delta V\left(\theta_{i}^{+}\right)\right|}=\frac{\Delta V\left(\theta_{+}\right)}{\left|\Delta V\left(\theta_{+}\right)\right|}=-\frac{\lambda_{1}}{\sqrt{1-e^{2}}}=\varepsilon .
$$

and for all $i=1, \cdots, N_{-}$,

$$
\frac{\Delta V\left(\theta_{i}^{-}\right)}{\left|\Delta V\left(\theta_{i}^{-}\right)\right|}=\frac{\Delta V\left(\theta_{-}\right)}{\left|\Delta V\left(\theta_{-}\right)\right|}=\frac{\lambda_{1}}{\sqrt{1-e^{2}}}=-\varepsilon .
$$

86) and (87) indicate that the optimal directions are alternating between $\theta_{i}^{+}$and $\theta_{i}^{-}$. Condition 14 leads to the following system of equations:

$$
\left[\begin{array}{c}
\sqrt{1-e^{2}} \\
e
\end{array}\right] \sum_{i=1}^{N_{+}}\left|\Delta V\left(\theta_{i}^{+}\right)\right|+\left[\begin{array}{c}
\sqrt{1-e^{2}} \\
-e
\end{array}\right] \sum_{i=1}^{N_{-}}\left|\Delta V\left(\theta_{i}^{-}\right)\right|
$$


from which may be easily computed the optimal consumption:

$$
\begin{gathered}
\sum_{i=1}^{N_{+}}\left|\Delta V\left(\theta_{i}^{+}\right)\right|=\varepsilon \frac{\sqrt{1-e^{2}}}{2 e}\left(e z_{f_{1}}+\sqrt{1-e^{2}} z_{f_{2}}\right), \\
\sum_{i=1}^{N_{-}}\left|\Delta V\left(\theta_{i}^{-}\right)\right|=\varepsilon \frac{\sqrt{1-e^{2}}}{2 e}\left(e z_{f_{1}}-\sqrt{1-e^{2}} z_{f_{2}}\right), \\
\sum_{i=1}^{N_{+}}\left|\Delta V\left(\theta_{i}^{+}\right)\right|+\sum_{i=1}^{N_{-}}\left|\Delta V\left(\theta_{i}^{-}\right)\right|=\varepsilon z_{f_{1}} \sqrt{1-e^{2}} .
\end{gathered}
$$

From the positive sign of (88) and (89), it is deduced that $\varepsilon=\operatorname{sgn}\left(z_{f_{1}}\right)=\varepsilon_{1}$. Then, $\left(e z_{f_{1}}+\sqrt{1-e^{2}} z_{f_{2}}\right)$ and $\left(e z_{f_{1}}-\sqrt{1-e^{2}} z_{f_{2}}\right)$ are necessarily not equal to 0 and have the same sign which is equivalent to $e\left|\vec{z}_{f}\right|>\left|z_{f_{2}}\right|$. Indeed, if $e\left|\vec{z}_{f}\right|=\left|z_{f_{2}}\right|$, the optimal solution comes down to one interior impulse solution as described in the next section, with at most one positive or negative impulse per period. The first optimal Lagrange multiplier is then obtained from (11) as $\lambda_{1}^{*}=-\varepsilon_{1} \sqrt{1-e^{2}}$. In conclusion, when $d_{\theta} \geq 2 \pi$, the optimal solution of the planning may be chosen to be concentrated over two impulses, as is presented in Proposition 1 or spread over $N_{-}+N_{+}$impulses verifying (88) and (89), depending on the duration of the rendezvous and operational constraints. Finally, the optimal consumption is defined by $\left|z_{f_{1}}\right| \sqrt{1-e^{2}}$.

Let now consider the case where the rendezvous lasts less than $2 \pi$ i.e. $d_{\theta}<2 \pi$. The discussion is very similar to the case where $\theta_{f}-\theta_{0} \geq 2 \pi$ and leads to the conditions $e\left|\vec{z}_{f}\right|>\left|z_{f_{2}}\right|$ and $\left(\theta_{+}, \theta_{-}\right) \in\left[\theta_{0}, \theta_{f}\right]^{2}$.

- Case $d_{\theta}<\pi$. There exists $\theta_{+}, \theta_{-} \in\left[\theta_{0}, \theta_{f}\right]$ such that $\cos \left(\theta_{ \pm}\right)=-e$ and $\sin \left(\theta_{ \pm}\right)= \pm \sqrt{1-e^{2}}$ if and only if:

$$
\sin \left(\theta_{0}\right) \geq \sqrt{1-e^{2}}, \quad \sin \left(\theta_{f}\right) \leq-\sqrt{1-e^{2}}
$$

- Case $\pi<d_{\theta}<2 \pi$. There exists $\theta_{+}, \theta_{-} \in\left[\theta_{0}, \theta_{f}\right]$ such that 91] if and only if

$$
\begin{aligned}
\sin \left(\theta_{0}\right) \geq \sqrt{1-e^{2}} & \text { or }\left\{\begin{array}{l}
\sin \left(\theta_{0}\right) \leq \sqrt{1-e^{2}} \\
\sin \left(\theta_{f}\right) \leq-\sqrt{1-e^{2}}
\end{array}\right. \\
& \text { or }\left\{\begin{array}{l}
\left|\sin \left(\theta_{0}\right)\right|<\sqrt{1-e^{2}} \\
\left(e+\cos \left(\theta_{0}\right)\right)\left(e+\cos \left(\theta_{f}\right)\right)>0 .
\end{array}\right.
\end{aligned}
$$

Under these conditions, the optimal solution is a 2-impulse solution defined by the optimal locations $\theta_{+}$and $\theta_{-}$and

$$
\begin{aligned}
\Delta V\left(\theta_{ \pm}\right) & =\frac{\sqrt{1-e^{2}}}{2 e}\left(\mp e z_{f_{1}}-\sqrt{1-e^{2}} z_{f_{2}}\right), \\
\lambda_{1}^{*} & =-\varepsilon_{1} \sqrt{1-e^{2}}, \quad \lambda_{2}^{*}=0 .
\end{aligned}
$$

In both cases the optimal primer vector is given by:

$$
p^{*}(\theta)=\varepsilon_{1} \frac{\sqrt{1-e^{2} \sin (\theta)}}{1+e \cos (\theta)} .
$$

\section{REFERENCES}

[1] T. Carter, "Optimal impulsive space trajectories based on linear equations," Journal of Optimization Theory and Applications, vol. 70, no. 2, pp. 277-297, August 1991, doi: 10.1007/BF00940627.

[2] T. Carter and J. Brient, "Linearized impulsive rendezvous problem," Journal of Optimization Theory and Applications, vol. 86, no. 3, pp. 553-584, September 1995, doi: 10.1007/BF02192159.

[3] J. Prussing, "Illustration of the primer vector in time-fixed orbit transfer," AIAA Journal, vol. 7, no. 6, pp. 1167-1168, 1969, doi: 10.2514/3.5297.
[4] L. Riggi and S. D'Amico, "Optimal impulsive closed-form control for spacecraft formation flying and rendezvous," in Proceedings of the American Control Conference, Boston, MA, USA, July 2016.

[5] C. Roscoe, J. Westphal, J. Griesbach, and H. Schaub, "Formation establishment and reconfiguration using differential elements in $j_{2}$ perturbed orbits," in Proceedings of the IEEE Aerospace Conference, Big Sky, MT, USA, March 2014.

[6] L. Neustadt, "Optimization, a moment problem, and nonlinear programming," SIAM Journal of Control, vol. 2, no. 1, pp. 33-53, 1964.

[7] R. Serra, D. Arzelier, A. Rondepierre, and J. Calvet, "Analytical optimal solutions of impulsive out-of-plane rendezvous around elliptic orbits," in Proceedings of the IFAC World Congress, Cape Town, South Africa, August 2014, pp. 2231-2236.

[8] R. Serra, D. Arzelier, and A. Rondepierre, "Analytical optimal solutions of impulsive out-of-plane rendezvous around elliptic orbits: Results and derivations," LAAS-CNRS, Technical Note 14143, April 2014, http://homepages.laas.fr/arzelier/publis/2014/HP-journal.pdf.

[9] J. Prussing, "Optimal multiple-impulse orbital rendezvous," Ph.D. dissertation, Massachusetts Institute of Technology, Cambridge, Massachusetts, 1967.

[10] J. Prussing and J. Chiu, "Optimal multiple-impulse time-fixed rendezvous between circular orbits," Journal of Guidance, vol. 9, no. 1, pp. 17-22, 1986.

[11] T. Carter and S. Alvarez, "Quadratic-based computation of four-imulse optimal rendezvous near circular orbit," Journal of Guidance, Control and Dynamics, vol. 23, no. 1, pp. 109-117, 2000, doi: 10.2514/2.4493.

[12] D. Arzelier, M. Kara-Zaitri, D. Louembet, and A. Delibasi, "Using polynomial optimization to solve the fuel-optimal linear impulsive rendezvous problem," Journal of Guidance, Control, and Dynamics, vol. 34, no. 5, pp. 1567-1576, 2011, doi: 10.2514/1.52227.

[13] D. Arzelier, D. Louembet, A. Rondepierre, and M. Kara-Zaitri, "A new mixed iterative algorithm to solve the fuel-optimal linear impulsive rendezvous problem," Journal of Optimization Theory and Applications, vol. 159, no. 1, pp. 210-230, 2013.

[14] K. Yamanaka and F. Ankersen, "New state transition matrix for relative motion on an arbitrary elliptical orbit," Journal of Guidance, Control and Dynamics, vol. 25, no. 1, january 2002, doi: 10.2514/2.4875.

[15] D. Lawden, Optimal trajectories for space navigation. London, England: Butterworth, 1963.

[16] J. Prussing, "Optimal impulsive linear systems: Sufficient conditions and maximum number of impulses," Journal of the Astronautical Sciences, vol. 43, no. 2, pp. 195-206, 1995.

[17] T. Carter, "Necessary and sufficient conditions for optimal impulsive rendezvous with linear equations of motion," Dynamics and Control, vol. 10, pp. 219-227, 2000, doi: 10.1023/A:1008376427023.

[18] J. Prussing, "Primer vector theory and applications," in Spacecraft Trajectory Optimization, B. Conway, Ed. New York, NY, USA: Cambridge University Press, 2010, pp. 16-36.

[19] J. Peyrard, D. Escorial, A. Agenjo, A. Kron, and A. Cropp, "Design and prototyping of proba-3 formation flying system," in Proceedings of the 5th International Conference on Spacecraft Formation Flying Missions and Technologies (SFFMT), Munich, Germany, May 2013.

[20] B. Zhou, Z. Lin, and G. Duan, "Lyapunov differential equation approach to elliptical orbital rendezvous with constrained controls," Journal of Guidance, Control and Dynamics, vol. 34, no. 2, pp. 345-358, 2011. 